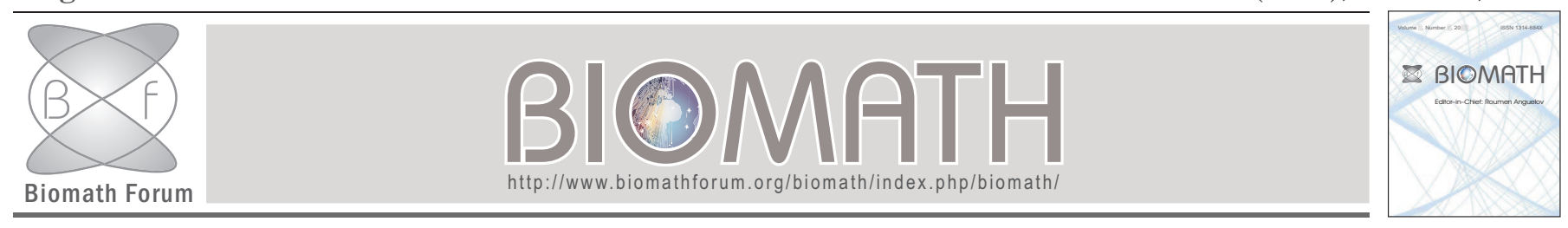

\title{
A patchy model for Chikungunya-like diseases
}

\author{
Samuel Bowong \\ University of Douala \\ Faculty of Science \\ UMI 209 IRD/UPMC UMMISCO \\ GRIMCAPE project team \\ Douala, Cameroon \\ sbowong@gmail.com
}

\author{
Yves Dumont \\ CIRAD \\ Umr AMAP 34392 \\ Montpellier, France \\ yves.dumont@cirad.fr
}

\author{
Jean Jules Tewa \\ National Advanced School of Engineering \\ University of Yaoundé I, \\ UMI 209 IRD/UPMC UMMISCO \\ GRIMCAPE project team \\ Yaoundé, Cameroon \\ tewajules@gmail.com
}

Received: 21 February 2013, accepted: 23 July 2013, published: 31 July 2013

\begin{abstract}
We consider a $n$-patches model, to study the impact of human population movements between cities (patches) in the spread of Chikungunya or even Dengue diseases. In previous works, it was showed that the basic reproduction number can vary from place to place, but this result was obtained without taking into account human movements. We provide a theoretical study of the patchy model, and derive $\mathcal{R}_{0}^{2}$, the basic reproduction number, which may depend on Human movement rates between the patches and on local population sizes. We show that $\mathcal{R}_{0}$ is bounded from above (below) by the maximum (minimum) of the values of the local basic reproduction numbers. We also show that there exists a disease-free equilibrium $\mathbf{E}_{D F}$ that is locally asymptotically stable whenever $\mathcal{R}_{0}^{2}<1$. Under suitable assumptions, $\mathbf{E}_{D F}$ is even globally asymptotically stable. We emphasize that Human movements are of particular importance to evaluate the spreading or not of Chikungunya or Dengue diseases, and thus movement rates have to be estimated very accurately. We confirm also the importance to know where local basic reproduction numbers are large and show that local field interventions can help to control/reduce the spread of the disease. A full analytical study for the 2-patches model and several simulations are provided to illustrate that human movements can either increase or reduce the spreading of the disease.
\end{abstract}

Keywords-Patch; Chikungunya; Dengue; Movements; Disease free equilibrium; Basic reproduction number; Endemic equilibrium; Local and Global Stability.

AMS Classification: 92-08, 92D30, 37M05, 65L12, $92 \mathrm{C60}$.

\section{INTRODUCTION}

Chikungunya is a vector-borne disease caused by Aedes albopictus. It is an uncommon and not well-known tropical disease whose dynamics and behaviour are yet to be fully understood [32]. A good understanding of its transmission dynamics and its ecology in emergent epidemic regions like Réunion Island can help to improve the control of future epidemics around the world. Mathematical models provide a quantitative and potentially valuable tool for this purpose. The ability to forecast, understand and control the spread of infectious diseases increasingly depends on the capacity to formulate and test mathematical models capturing key mechanisms. The present study builds on and extend previous works on the Chikungunya disease [18], [19], [22].

Chikungunya is endemic in East Africa and in Asia. The main symptoms are fever, headache and arthritis, that can lead to severe clinical cases, and sometimes, deaths [30]. It appeared in developed countries, like Réunion Island, in 2005 and 2006, in Italy and India, in 2007, and recently in Congo-Brazaville, in 2011. Two cases have also been reported in September 2010 in the South-East of France. The principal vector of the Chikungunya in Réunion Island and in Italy is Aedes albopictus (sometimes called the Asian tiger because it originated from Asia and it is an agressive mosquito), which is also a prospective vector for Dengue transmission. In a recent period, there has been a tremendous 
progress in our knowledge about the vector and the relationships between the virus and the vector (see, for instance, [12], [13], [14], [15], [27], [34], [35]). One of the first models for the Chikungunya epidemic of 20052006 in Réunion Island was proposed in [18]. The focus in [19] was on the study on chemical and mechanical tools available to stop or to control an epidemic, where it is shown that the combination of Deltamethrin, the only authorized adulticide in the European Union, and mechanical control, which consists in reducing the breeding sites, could have been useful to stop the huge epidemic of 2006. Recently, a study has been done on the Sterile Insect Technique as a potential vector control tool for the Chikungunya Disease [22].

Another very important point is that two strains of the virus were isolated in Réunion Island. The first one, strain 05.115, was isolated in May 2005, during the first outbreak, and the second one, strain 06.21, was isolated later, mid November 2005 (in fact we don't know exactly when the mutation happened). Vazeille et al. proved that strain 06.21 had a larger rate of transmission from human to mosquito [34]. In [18], the authors were the first to take into account this assumption. Their numerical simulations showed that strain 06.21 was certainly responsible of the explosive epidemic from 2006. Moreover strain 06.21 had a direct impact on the lifespan of infected mosquitoes [27]. It is an unusual assumption (usually, in vector-borne disease models, the mean mortality rate of the mosquito, in the different epidemiological states, is assumed to be constant) that makes the theoretical analysis of the model more difficult [19], [22]. In this paper, we will only consider one strain, to simplify the analysis.

Since a couple of years, metapopulation models have been studied a lot, in particular to understand the dynamics of infectious diseases [7], [25]. In [25], the authors have revisited how metapopulation processes operate at various spatial scales (individual level, local, and regional epidemics). They have illustrated the resultant spatio-temporal dynamics by a series of case studies which explore diseases metapopulation dynamics at the interface of models and data. However, the mathematical analysis of the model (existence and stability of equilibria) has not been done in their studies. More recently, metapopulation or patch models have been applied to Malaria disease [8], [23] indicating clearly that human population movement is an important component to understand the time course of an epidemic.

In [18], [19], the studies only focused on local places, in order to detect where the epidemiological risk is high, in other words, where the basic reproduction number is greater than one. In recent works [20], [21], [16], [17], spatio-temporal models, using partial differential equations, have been developed to study mosquito displacements according to landscape elements. In [17], the authors took into account environmental factors, like temperature, to study different SIT control strategies, taking into account periodic releases of steriles males.

The aim of this work is now to link the cities, taking into account human movements, that could explain the spread of the disease. For instance, in Réunion Island, in 2005, the first Chikungunya case was referenced in Saint-Pierre, the 22th of February 2005, the next in SaintDenis and, then in La Possession, and Le Port.... Using temporal (and even spatio-temporal) data from the ARS (Regional French Health agency) in Réunion Island, we know about the time and spatial spread of the epidemic. In Réunion Island, the car is the favorite transportation and people travel a lot from place to place in the island to go from Home to work and back or to visit family or friends. Moreover, it is well known that due to arthritis many infected and even recovered (not infectious) people were not able to move [30]. Thus, in our model, we intend to take into account limitation movements of infected populations and show that it can have an impact in the spread and the force of the epidemic. Of course, a possible and nice extension of our model would be to consider the different Islands in Indian Ocean, like Mauritius, la Réunion, Comoros, and Madagascar as a possible 4 patches model.

The outline of the paper is as follows: in section 2, we present the migration model and the full epidemiological model for $n$ cities. In section 3 , we compute the DFE, and the general basic reproduction number, $\mathcal{R}_{0}^{2}$, and show that the DFE is locally asymptotically stable (LAS). Then we show that the DFE can sometimes be globally asymptotically stable. In section 4 , we study the spreading of the disease. Finally, we validate our theoretical results with a two patches model.

\section{A. The human migration model}

Our study focus on four cities in Réunion Island, but we present the migration model in a general setting, in order to have a generic modeling. All cities are more or less connected, and, in principle, people can move from one town to another. We assume that the total population is constant. In Figure 1, we present an example of a npatches model: each city is a patch. In this figure, the solid line stand from one city to another which means that there is a strong connection between the cities, while 
the dotted line is from one city to another which means that the connection is weak.

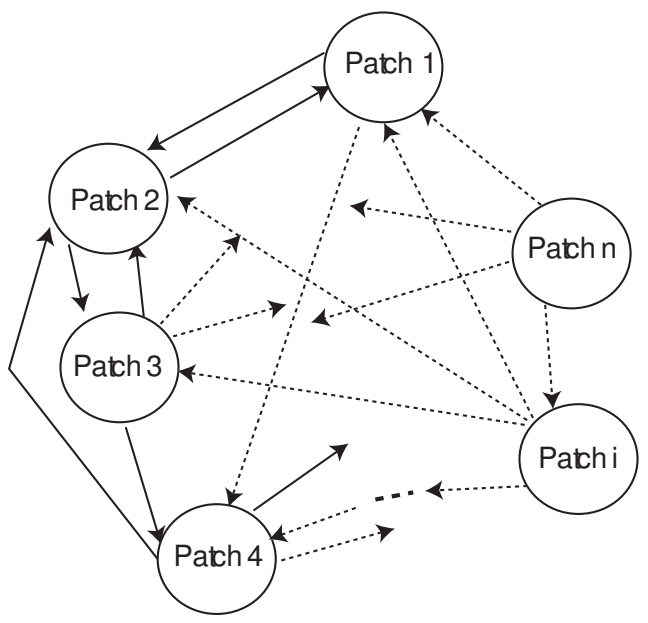

Fig. 1. A general n-patches model for the transmission of the Chikungunya virus between $n$ cities in Réunion Island.

Assuming that the total human population in each patch is denoted by $N_{i, h}$, we have $N_{i, h}=S_{i, h}+I_{i, h}+$ $R_{i, h}$, for $i=1, \ldots, n$. Moreover, the total population $N_{t o t}$ verifies $N_{t o t}=N_{1, h}+\cdots+N_{i, h}+\cdots+N_{n, h}$.

For each epidemiological states, we consider the following migration model:

$$
\frac{d X_{i, h}}{d t}=\sum_{\substack{j=1 \\ j \neq i}}^{n} m_{i j}^{X_{h}} X_{j, h}-\left(\sum_{\substack{j=1 \\ j \neq i}}^{n} m_{j i}^{X_{h}}\right) X_{i, h},
$$

where $X_{i, h} \in\left\{S_{i, h}, I_{i, h}, R_{i, h}\right\}$. Now, setting $X_{h}=$ $\left(X_{1, h}, \ldots, X_{n, h}\right)^{T}$, the migration model (1) becomes

$$
\begin{aligned}
& \frac{d X_{h}}{d t}=\mathcal{M}^{X_{h}} X_{h}, \\
& \text { where } \mathcal{M}^{X_{h}}=\left(\begin{array}{ccc}
\mathcal{M}_{1,1} & \ldots & \mathcal{M}_{1, n} \\
\ldots & \ldots & \ldots \\
\mathcal{M}_{n, 1} & \ldots & \mathcal{M}_{n, n}
\end{array}\right) \text {, with }
\end{aligned}
$$

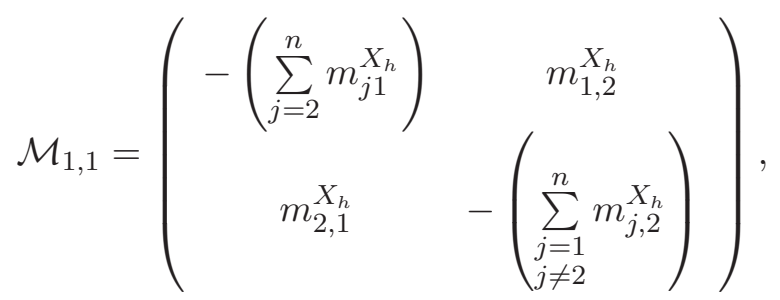

$$
\begin{aligned}
& \mathcal{M}_{1, n}=\left(\begin{array}{ll}
m_{1, n-1}^{X_{h}} & m_{1, n}^{X_{h}} \\
m_{2, n-1}^{X_{h}} & m_{2, n}^{X_{h}}
\end{array}\right), \\
& \mathcal{M}_{n, 1}=\left(\begin{array}{cc}
m_{n-1,1}^{X_{h}} & m_{n-1,2}^{X_{h}} \\
m_{n, 1}^{X, 1} & m_{n, 2}^{X_{h}}
\end{array}\right) \text {, }
\end{aligned}
$$

$$
\mathcal{M}_{n, n}=\left(\begin{array}{cc}
-\left(\begin{array}{c}
\sum_{\substack{j=1 \\
j \neq n-1}}^{n} m_{j, n-1}^{X_{h}} \\
m_{n, n-1}^{X_{h}}
\end{array}\right. & -\left(\sum_{n-1, n}^{X_{h}} \sum_{j=1}^{n-1} m_{j, n}^{X_{h}}\right)
\end{array}\right) .
$$

The coefficients $m_{i, j}^{X_{h}}$ are chosen according to the movement of the epidemiological state $X_{h}$ between the cities and with the constraints that a possible equilibrium of the total population $N_{t o t}$ corresponds to the inhabitants in each city.

We point out that $\mathcal{M}^{X_{h}}$ is a Metzler matrix, i.e. a matrix with all off-diagonal terms nonnegative, and is irreducible [9]. Thus, if $X_{h}(0) \in \mathbb{R}_{+}^{n}$, then $X_{h}(t) \in \mathbb{R}_{+}^{n}$. Indeed, there exist a real $s>0$ and a matrix $B \geq 0$ such that

$$
-\mathcal{M}^{X_{h}}=s I_{d_{n}}-B
$$

which implies that $-\mathcal{M}$ is a singular irreducible $\mathrm{M}$ matrix of order $n$. Thus, following Theorem 4.16, page 156 in [9], there exists a positive vector $L_{h}>>0$ such that

$$
\mathcal{M}^{X_{h}} L=0 \quad \text { with } \quad \sum_{i=1}^{n} L_{i, h}=1 .
$$

From Perron-Frobenius theorem [9], we deduce that there exists a simple eigenvalue $\lambda_{\max }=$ $\max _{\lambda \in \operatorname{sp}\left(\mathcal{M}^{x_{h}}\right)} \mathcal{R} e(\lambda)$ and an eigenvector $\omega_{h}>>0$ such that

$$
\mathcal{M}^{X_{h}} w_{h}=\lambda_{\max } w_{h}
$$

Now, using the fact that $\mathbf{1}^{T} \mathcal{M}^{X_{h}}=0$, where $\mathbf{1}^{T}=$ $(1,1, \ldots, 1)$, we have $\lambda_{\max }=0$, and thus all other eigenvalues have a negative real part. Thus, the following result hold.

Proposition 1: Equation (2) admits a unique positive equilibrium $X_{h}^{0}=X_{\text {tot }}^{0}$ which is globally asymptotically stable on the hyperplane orthogonal to $\mathbf{1}^{T}$.

\section{B. The full epidemiological model for $n$ cities.}

For each city we have temporal data, and some of them have been studied independently in Refs. [18], [19]. Our aim is to consider an epidemiological model in each patch and to take into account human movement between the patches. In patch $i$, we assume that the human population is constant and equal to $N_{i, h}$, and is subdivided in three compartmental stages : the susceptible, $S_{i, h}$, the infected $I_{i, h}$ and the recovered, $R_{i, h} . \mu_{i, h}$ is the per capita death rate of humans in susceptible, infectious and recover stages in patch $i$; this parameter 
is also assumed to be the recruitment rate of humans in the susceptible compartment stage, proportionally to the human population. In a same manner, we assume that all patches have got mosquitoes and, we consider three stages for the mosquitoes : an aquatic stage, $A_{i, m}$, the susceptible, $S_{i, m}$, and the Infected $I_{i, m} . K_{i}$ is the carrying capacity of all breeding sites, and $\mu_{i, b}$ is the number of eggs layed per day and per (female) mosquito, in patch $i$. An infected mosquito in patch $i$ can only infect a susceptible human from patch $i$. In each patch, we assume that mosquitoes and humans are homogeneously distributed. The aquatic state includes the eggs, larvae and pupae. Both humans and mosquitoes are assumed to be born susceptible. $\eta_{i, h}$ is the recovering rate of infected human in patch $i$ such that an infected human is infectious during $\frac{1}{\eta_{i, h}}$ days, called the viremic period, and then becomes resistant or immune. The parameter $\alpha_{i}$ is related to the carrying capacity and represents the level of mechanical control in patch $i$ : when $\alpha_{i}=1$, there is no mechanical control; when $\alpha_{i}=0.5$, it indicates that 50 percents of the breedings have been removed in patch $i . \mu_{i, A}$ is the per capita death rate of mosquitoes in aquatic stage in patch $i ; \mu_{i, m}$ is the per capita death rate of mosquitoes in susceptible and infectious stages in patch $i ; \eta_{i, A}$ is the rate of mosquitoes of patch $i$ which leave the aquatic stage and progress to the susceptible stage.

Cross-infection between humans and vectors is modeled by the mass-action principle normalized by the total population of humans. Every day, in patch $i$, each mosquito bites, on average, $B_{i}$ times. $p_{i, m h}$ is the probability that a bite on a susceptible individual will lead to host infection so that $\beta_{i, m h}=B_{i} p_{i, m h}$ represents the contact rate between infectious mosquitoes and susceptible hosts. Similarly, $\beta_{i, h m}=B_{i} p_{i, h m}$ is the contact rate between infectious hosts and susceptible mosquitoes, where $p_{i, h m}$ is the probability that a bite on an infected individual will lead to vector infection.

In Réunion Island, $80 \%$ of the population being living at the sea level, we assume that the parameters in the human compartments are the same in each patch (at least from Saint-Denis to Saint-Pierre). In patch $i$, we assume that the average lifespan for susceptible and infected mosquitoes is $1 / \mu_{i, m}$. Let us recall also, that in Réunion Island, it was proved that a mutation in the initial strain leads to a new strain that influences the lifespan of the infected mosquito: it is almost halved [27]. This uncommon result can influence the dynamics of the disease [19]. Here, for sake of simplicity in the analysis, we will only consider one strain (no mutation). For other vector-borne diseases it has never been observed that a mutation in the virus influences the lifespan of an infected mosquito. There is no evidence of vertical transmission [35]. We also assume that the mosquito parameters may change from patch to patch.

Note also that we don't consider the "exposed" stage, like in [18], [19], for sake of simplicity. All together, for $i=1, \ldots, n$, we have the following system for the mosquitoes population:

$$
\left\{\begin{array}{l}
\frac{d A_{i, m}}{d t}=\mu_{i, b}\left(1-\frac{A_{i, m}}{\alpha_{i} K_{i}}\right)\left(S_{i, m}+I_{i, m}\right) \\
\quad-\left(\eta_{i, A}+\mu_{i, A}\right) A_{i, m}, \\
\frac{d S_{i, m}}{d t}=-\beta_{i, h m}\left(\frac{I_{i, h}}{N_{i, h}}\right) S_{i, m}-\mu_{i, m} S_{i, m} \\
\quad+\eta_{i, A} A_{i, m}, \\
\frac{d I_{i, m}}{d t}=\beta_{i, h m}\left(\frac{I_{i, h}}{N_{i, h}}\right) S_{i, m}-\mu_{i, m} I_{i, m},
\end{array}\right.
$$

and the following differential system for the human population:

$$
\left\{\begin{array}{c}
\frac{d S_{i, h}}{d t}=\mu_{i, h} N_{i, h}-\beta_{i, m h} \frac{I_{i, m}}{N_{i, h}} S_{i, h}-\mu_{i, h} S_{i, h} \\
+\sum_{\substack{j=1 \\
j \neq i}}^{n} m_{i j}^{S} S_{j, h}-\left(\sum_{\substack{j=1 \\
j \neq i}}^{n} m_{j i}^{S}\right) S_{i, h}, \\
\frac{d I_{i, h}}{d t}=\beta_{i, m h} \frac{I_{i, m}}{N_{i, h}} S_{i, h}-\left(\mu_{i, h}+\eta_{i, h}\right) I_{i, h} \\
+\sum_{\substack{j=1 \\
j \neq i}}^{n} \gamma_{j} m_{i j}^{I} I_{j, h}-\gamma_{i}\left(\sum_{\substack{j=1 \\
j \neq i}}^{n} m_{j i}^{I}\right) I_{i, h}, \\
\frac{d R_{i, h}}{d t}=\eta_{i, h} I_{i, h}-\mu_{i, h} R_{i, h}+\sum_{\substack{j=1 \\
j \neq i}}^{n} m_{i j}^{R} R_{j, h} \\
-\left(\begin{array}{l}
\sum_{j=1}^{n} m_{j i}^{R} \\
j \neq i
\end{array}\right) R_{i, h},
\end{array}\right.
$$

where $S_{i, h}+I_{i, h}+R_{i, h}=N_{i, h}$. In addition, we have the following initial conditions in each patch: $\left(\alpha_{i} K_{i} ; m_{i} N_{i, h} ; 0 ; N_{i, h}-I_{i, h}^{0} ; I_{i, h}^{0} ; 0\right)$, where $I_{i, h}^{0}$ is the initial number of infected people in patch $i$, and $m_{i}$ a positive real number. In numerical simulations, we will consider that $K_{i}=k_{i} N_{i, h}$, where $k_{i}$ is a positive real number.

Setting $S_{h}=\left(S_{1, h}, \ldots, S_{n, h}\right), I_{h}=\left(I_{1, h}, \ldots, I_{n, h}\right)$, $R_{h}=\left(R_{1, h}, \ldots, R_{n, h}\right), A_{m}=\left(A_{1, m}, \ldots, A_{n, m}\right), S_{m}=$ $\left(S_{1, m}, \ldots, S_{n, m}\right)$ and $I_{m}=\left(I_{1, m}, \ldots, I_{n, m}\right)$. 
In the sequel, we will assume that the migration models for the epidemiological states $S_{h}$ and $R_{h}$ are the same, i.e. $\mathcal{M}^{S_{h}}=\mathcal{M}^{R_{h}}=\mathcal{M}$. However, Chikungunya fever is general symmetric with joint pains that occur in wrists, elbows, fingers, knees,..., leading sometimes to arthritis [31] such that it can be very difficult to drive and thus, going from one city to another and cannot always be possible for infected people. Thus, we will assume that $\mathcal{M}^{I_{h}}=\mathcal{M} \Gamma$, with $\Gamma=\operatorname{diag}\left(\gamma_{i}\right)$, where $\gamma_{i} \in[0,1]$; $\gamma_{i}$ indicates the proportion of infected people that were able to move from patch $i$ to the other patches. We could also assume that $\mathcal{M}^{R_{h}}=\mathcal{M} \Gamma$ too, since this joint problems can persist several weeks or months after the people had become viremic, but this hypothesis do not change the rest of the paper mainly because recovered people do not become susceptible again. So for sake of simplicity, we keep $\mathcal{M}^{R_{h}}=\mathcal{M}$ as assumed previously.

Remark 1: Our migration model doesn't take into account the home of the individuals, which would imply a far more complex model. In our modelling, we don't take into account people that moves daily, for instance from home to work and back. Indeed, ae. albopictus is only active early in the morning and late in the afternoon, thus more or less outside the office hours in Réunion island. Thus, we only consider people that stay more than one day, and thus have more or less the same probability than local people to be bitten. This is why we don't make distinction in a patch between people coming from different patches.

Therefore, in terms of $S_{h}, I_{h}, R_{h}, A_{m}, S_{m}$ and $I_{m}$, the differential equations (3) and (4) can be rewritten in the following vectorial form:

$$
\left\{\begin{array}{l}
\frac{d A_{m}}{d t}=\operatorname{diag}\left(\mu_{b}\right) \operatorname{diag}(K)^{-1} \operatorname{diag}\left(K-\frac{A_{m}}{\alpha}\right) S_{m} \\
+\operatorname{diag}\left(\mu_{b}\right) \operatorname{diag}(K)^{-1} \operatorname{diag}\left(K-\frac{A_{m}}{\alpha}\right) I_{m} \\
\quad-\operatorname{diag}\left(\eta_{A}+\mu_{A}\right) A_{m} \\
\frac{d S_{m}}{d t}=-\operatorname{diag}\left(N_{h}\right)^{-1} \operatorname{diag}\left(\beta_{h m} I_{h}\right) S_{m} \\
\quad-\operatorname{diag}\left(\mu_{m}\right) S_{m}+\operatorname{diag}\left(\eta_{A}\right) A_{m} \\
\frac{d I_{m}}{d t}=\operatorname{diag}\left(N_{h}\right)^{-1} \operatorname{diag}\left(\beta_{h m} I_{h}\right) S_{m}-\operatorname{diag}\left(\mu_{m}\right) I_{m},
\end{array}\right.
$$

and

$$
\left\{\begin{array}{l}
\frac{d S_{h}}{d t}=\mu_{h} I d_{n}\left(I_{h}+R_{h}\right) \\
\quad-\operatorname{diag}\left(N_{h}\right)^{-1} \operatorname{diag}\left(\beta_{m h} I_{m}\right) S_{h}+\mathcal{M} S_{h}, \\
\begin{array}{rl}
\frac{d I_{h}}{d t} & =\operatorname{diag}\left(N_{h}\right)^{-1} \operatorname{diag}\left(\beta_{m h} I_{m}\right) S_{h} \\
& -\left(\eta_{h}+\mu_{h}\right) I d_{n} I_{h}+\mathcal{M} \Gamma I_{h}
\end{array} \\
\frac{d R_{h}}{d t}=\eta_{h} I d_{n} I_{h}-\mu_{h} I d_{n} R_{h}+\mathcal{M} R_{h}
\end{array}\right.
$$

where $\mu_{b}=\left(\mu_{1, b}, \cdots, \mu_{n, b}\right)^{T}, K=\left(K_{1}, \cdots, K_{n}\right)^{T}$, $\alpha=\left(\alpha_{1}, \cdots, \alpha_{n}\right)^{T}, \eta_{A}=\left(\eta_{1, A}, \cdots, \eta_{n, A}\right)^{T}, \mu_{A}=$ $\left(\mu_{1, A}, \cdots, \mu_{n, A}\right)^{T}, \beta_{h m}=\left(\beta_{1, h m}, \cdots, \beta_{n, h m}\right)^{T}, \mu_{m}=$ $\left(\mu_{1, m}, \cdots, \mu_{n, m}\right)^{T}$ and $\operatorname{diag}(Y)$ denotes the diagonal matrix of order $n$ defined by the vector $Y$ of ${ }^{n}$.

Summing sub-systems $\left({ }_{6}\right)_{1},(6)_{2}$, and $(6)_{3}$ gives $\frac{d N_{h}}{d t}=\mathcal{M} S_{h}+\mathcal{M} \Gamma I_{h}+\mathcal{M} R_{h}=\mathcal{M} N_{h}-\mathcal{M}\left(I d_{n}-\Gamma\right) I_{h}$.

Then, the coupled system (5)-(6) may be rewritten in the following compact form:

$$
\left\{\begin{array}{l}
\frac{d V_{m}}{d t}=\mathcal{A}\left(H, V_{m}\right) V_{m} \\
\frac{d H}{d t}=\mathcal{B}\left(H, V_{m}\right) H
\end{array}\right.
$$

where

$$
\begin{aligned}
& H=\left(S_{1, h}, \cdots, S_{n, h}, I_{1, h}, \cdots, I_{n, h}, R_{1, h}, \cdots, R_{n, h}\right)^{T}, \\
& V_{m}=\left(A_{1, m}, \cdots, A_{n, m}, S_{1, m}, \cdots, S_{n, m}, I_{1, m}, \cdots, I_{n, m}\right)^{T},
\end{aligned}
$$$$
\mathcal{B}\left(H, V_{m}\right)=\left(\begin{array}{ccc}
-b_{11} & \mu_{h} I d_{n} & \mu_{h} I d_{n} \\
b_{21} & -b_{22} & 0 \\
0 & \eta_{h} I d_{n} & -\left(\mu_{h} I d_{n}-\mathcal{M}\right)
\end{array}\right),
$$

with $b_{11}=\left(\operatorname{diag}\left(N_{h}\right)^{-1} \operatorname{diag}\left(\beta_{m h} I_{m}\right)-\mathcal{M}\right)$, $b_{21}=\operatorname{diag}\left(N_{h}\right)^{-1} \operatorname{diag}\left(\beta_{m h} I_{m}\right), \quad b_{22}=$ $\left(\left(\eta_{h}+\mu_{h}\right) I d_{n}-\Gamma \mathcal{M}\right)$,

$$
\mathcal{A}\left(H, V_{m}\right)=\left(\begin{array}{ccc}
-\mathcal{A}_{11} & \operatorname{diag}\left(\mu_{b}\right) & \operatorname{diag}\left(\mu_{b}\right) \\
\operatorname{diag}\left(\eta_{A}\right) & -\mathcal{A}_{22} & 0 \\
0 & a_{32} & -\operatorname{diag}\left(\mu_{m}\right)
\end{array}\right),
$$

where $I d_{n}$ denote the identity matrix of ${ }^{n}, \mathcal{A}_{11}=$ $\left(\operatorname{diag}\left(\mu_{b}\right) \operatorname{diag}(\alpha K)^{-1}\left(S_{m}+I_{m}\right)+\operatorname{diag}\left(\eta_{A}+\mu_{A}\right)\right)$ and $\mathcal{A}_{22}=\left(\operatorname{diag}\left(N_{h}\right)^{-1} \operatorname{diag}\left(\beta_{h m} I_{h}\right)+\operatorname{diag}\left(\mu_{m}\right)\right)$, $a_{32}=\operatorname{diag}\left(\beta_{h m}\right) \operatorname{diag}\left(N_{h}\right)^{-1} \operatorname{diag}\left(I_{h}\right)$.

Note that $\mathcal{A}\left(H, V_{m}\right)$ and $\mathcal{B}\left(H, V_{m}\right)$ are Metzler matrices for all $V_{m} \in \mathbb{R}_{+}^{3 n}$ and all $H \in \mathbb{R}_{+}^{3 n}$. Thus, system (8) is positively invariant in $\mathbb{R}_{+}^{3 n} \times \mathbb{R}_{+}^{3 n}$, which means 
that any trajectory of the system starting from an initial state in the positive orthant $\mathbb{R}_{+}^{3 n} \times \mathbb{R}_{+}^{3 n}$ remains forever in $\mathbb{R}_{+}^{3 n} \times \mathbb{R}_{+}^{3 n}$. Note also that the right-hand side of system (8) being Lipschitz continuous so that there exists a unique maximal solution.

Now, let us show that the solutions are bounded. Using equation (7) and the fact that $\Gamma \leq I d_{n}$ gives

$$
\frac{d N_{h}}{d t} \leq \mathcal{M} N_{h} \text {. }
$$

Since $\sum_{i=1}^{n} \mathcal{M}_{i j}=0$, for all $j$, the total population $N_{\text {tot }}=\sum_{i=1}^{n} N_{i, h}$ in the full system is bounded by the initial total population $N_{t o t}^{0}=N_{1, h}^{0}+\cdots+N_{n, h}^{0}$, which implies that $N_{i, h}$, the total population in a given patch $i$, is also bounded by $N_{i, h}^{0}$. Thus, $S_{h}, I_{h}$ and $R_{h}$ are such that $\left(S_{h}, I_{h}, R_{h}\right) \leq\left(I d_{n}, I d_{n}, I d_{n}\right) N_{h}^{0}$, where $N_{h}^{0}=\left(N_{1, h}^{0}, \cdots, N_{n, h}^{0}\right)^{T}$.

In each patch, the basic offspring number related to the mosquitoes population is defined by

$$
\mathcal{N}_{i, m}=\frac{\mu_{i, b} \eta_{i, A}}{\left(\mu_{i, A}+\eta_{i, A}\right) \mu_{i, m}} .
$$

Without infectious mosquitoes and infectious humans, the mosquito dynamical system in each path $i$ reduces to

$$
\left\{\begin{array}{l}
\frac{d A_{i, m}}{d t}=\mu_{i, b}\left(1-\frac{A_{i, m}}{\alpha_{i} K_{i}}\right) S_{i, m}-\left(\eta_{i, A}+\mu_{i, A}\right) A_{i, m}, \\
\frac{d S_{i, m}}{d t}=-\mu_{i, m} S_{i, m}+\eta_{i, A} A_{i, m} .
\end{array}\right.
$$

System (10) has two equilibria $E_{0}=(0,0)$ and, when $\mathcal{N}_{i, m}>1, E_{i}^{\#}=\left(A_{m}^{0}, S_{m}^{0}\right)$, with

$A_{m}^{0}=\left(1-\frac{1}{\mathcal{N}_{i, m}}\right) \alpha_{i} K_{i}, S_{m}^{0}=\frac{\eta_{i, A}}{\mu_{i, m}}\left(1-\frac{1}{\mathcal{N}_{i, m}}\right) \alpha_{i} K_{i}\left\{\begin{array}{l}\dot{A}_{i, m}=\mu_{i, b}\left(1-\frac{A_{i, m}}{\alpha_{i} K_{i}}\right) S_{i, m}-\left(\eta_{i, A}+\mu_{i, A}\right) A_{i, m}, \\ \dot{S}_{i, m}+\dot{I}_{i, m}=\eta_{i, A} A_{i, m}-\mu_{i, m}\left(I_{i, m}+S_{i, m}\right) .\end{array}\right.$

In fact, using [5], we can show the following

Theorem 1: Let $A_{i, m}(0) \leq \alpha_{i} K_{i}$, then the following results hold

1) System (10) defines a cooperative dissipative dynamical system on $\mathbb{R}_{+}^{2}$.

2) If $\mathcal{N}_{i, m} \leq 1$, then the equilibrium $E_{0}$ is globally asymptotically stable on $\mathbb{R}_{+}^{2}$.

3) If $\mathcal{N}_{i, m}>1$, then system (10) has two equilibria $E_{0}$ and $E^{\#}$, where $E_{0}$ is unstable and $E^{\#}$ is stable

\section{Proof:} with basin of attraction $\mathbb{R}_{+}^{2} \backslash\left\{E_{0}\right\}$.

1) This is straightforward to verify.

2) It suffices to use (1) and to verify the assumptions of Theorem 5 given in Appendix A (see also [5]) with $\mathbf{a}=E_{0}$ and $\mathbf{b}=\left(\alpha_{i} K_{i}, \frac{2 \alpha_{i} \eta_{i, A}}{\mu_{i, m}} K_{i}\right)$.
3) Since $\mathcal{N}_{i, m}>1$, the inequality

$$
\mathcal{N}_{i, m}>\frac{1+C}{1-\frac{C}{\alpha_{i} K_{i}}}
$$

holds for $C>0$ sufficiently small. Let $\varepsilon>0$ and $A_{\varepsilon}$ be so small that we verify the previous inequality and the following ones: $A_{i, m, \varepsilon} \leq \varepsilon$ and $S_{i, m, \varepsilon}=\frac{\eta_{i, A}}{\mu i, m} \frac{\varepsilon}{1+\varepsilon} \leq \varepsilon$. Then the right-hand side of (10), taken at $\mathbf{a}_{\varepsilon}=\left(A_{i, m, \varepsilon}, S_{i, m, \varepsilon}\right)$ is equal to

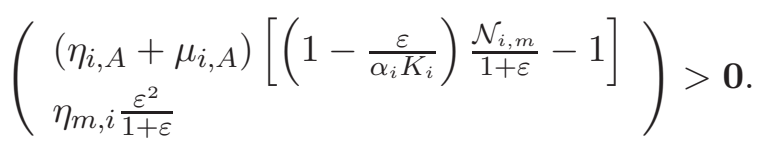

Thus applying Theorem [5, we deduce that $E^{\#}$ is GAS on $\left[\mathbf{a}_{\varepsilon}, \mathbf{b}_{m}\right]$. Since $\mathbf{a}_{\varepsilon}\left(\mathbf{b}_{m}\right)$ can be chosen smaller (larger) as needed, we deduce that $E^{\#}$ is asymptotically stable in $\mathbb{R}_{+}^{2}$ with basin of attraction at least the interior of $\mathbb{R}_{+}^{2}$. It can be easily seen that system (10) is irreducible. Let $z \in \mathbb{R}_{+}^{2} \backslash\left\{E_{0}\right\}$, that is, $z>\mathbf{0}$. Then it follows from a strong version of Kamke's Theorem (see Theorem 4 [5]) that $E_{i}(t)=\left(A_{i}(z, t), S_{i}(z, t)\right) \gg \mathbf{0}$ for $t>0$. Hence we have $\lim _{t \rightarrow+\infty} E_{i}(z, t)=E^{\#}$. Thus, the basin of attraction of $E^{\#}$ is precisely $\mathbb{R}_{+}^{2} \backslash\left\{E_{0}\right\}$. This also implies that $E_{0}$ is unstable, which completes the proof.

Finally, in patch $i$, from equations $(3)_{2}$ and $(3)_{3}$, we derive

Then, straightforward computations show that in each patch $i$, we have

$$
\begin{aligned}
& A_{i, m}(t) \leq A_{i, \max }=\max \left(A_{i, m}(0), \alpha_{i} K_{i}\right), \\
& S_{i, m}(t)+I_{i, m}(t) \leq \max \left(S_{i, m}(0)+I_{i, m}(0), \frac{\eta_{i, A} A_{i, \max }}{\mu_{i, m}}\right) .
\end{aligned}
$$

Therefore, the following theorem hold.

Theorem 2: Setting $H=\left(S_{h}, I_{h}, R_{h}\right)$ and $V_{m}=$ $\left(A_{m}, S_{m}, I_{m}\right)$. System (5)-(6) is invariant in $B=$

$$
\left\{\begin{array}{l}
\left(V_{m}, H\right) \in \mathbb{R}_{+}^{3 n} \times \mathbb{R}_{+}^{3 n} \mid S_{h}+I_{h}+R_{h}=N_{h}^{0}, \\
A_{m} \leq A_{\max } \\
S_{m}+I_{m} \leq \max \left(S_{m}(0)+I_{m}(0), \frac{\eta_{i, A}}{\mu_{i, m}} A_{\max }\right)
\end{array}\right\} .
$$




\section{DISEASE-FREE EQUILIBRIUM AND BASIC REPRODUCTION NUMBER}

\section{A. The disease-free equilibrium}

We consider systems (5) and (6) together. At the disease-free equilibrium (DFE), $I_{h}=0$ and $I_{m}=0$. Then, system (5) and (6) at the DFE gives

$$
\left\{\begin{array}{l}
-\mu_{h} N_{h}^{0}=\left(-\mu_{h} I d_{n}+\mathcal{M}\right) S_{h}^{0}, \\
\left(-\mu_{h} I d_{n}+\mathcal{M}\right) R_{h}^{0}=0, \\
\operatorname{diag}\left(\mu_{b}\right) \operatorname{diag}(K)^{-1} \operatorname{diag}\left(K-\frac{A_{m}^{0}}{\alpha}\right) S_{m}^{0} \\
=\operatorname{diag}\left(\eta_{A}+\mu_{A}\right) A_{m}^{0}, \\
\mu_{m} S_{m}^{0}=\eta_{A} A_{m}^{0} .
\end{array}\right.
$$

- Using Proposition 11, and choosing $S_{h}^{0}=N_{h}^{0}$, Eq. $(12)_{1}$ is verified.

- Equation $(12)_{2}$ implies that $R_{h}^{0}=0$. Also, from Eq. (12) 3 , one has

$$
\begin{aligned}
& \operatorname{diag}\left(\mu_{b}\right) \operatorname{diag}(K)^{-1} \operatorname{diag}\left(K-\frac{A_{m}^{0}}{\alpha}\right) S_{m}^{0} \\
& =\operatorname{diag}\left(\eta_{A}+\mu_{A}\right) A_{m}^{0} .
\end{aligned}
$$

Since $\mu_{i, m} S_{i, m}^{0}=\eta_{i, A} A_{i, m}^{0}$, for each $i$, the above equation becomes

$$
\begin{aligned}
& \operatorname{diag}\left(\mu_{b}\right) \operatorname{diag}(K)^{-1} \operatorname{diag}\left(K-\frac{A_{m}^{0}}{\alpha}\right) \operatorname{diag}\left(\eta_{A}\right) \\
& \times\left(\operatorname{diag}\left(\mu_{m}\right)\right)^{-1} A_{m}^{0}=\operatorname{diag}\left(\eta_{A}+\mu_{A}\right) A_{m}^{0} .
\end{aligned}
$$

Then, in each patch, there are two possibilities : $A_{i, m}^{0}=S_{i, m}^{0}=0$, or $A_{i, m}^{0}>0$ and $S_{i, m}^{0}>0$, depending on the value taken by the basic offspring number $\mathcal{N}_{i, m}$ (see the previous computations above).

Finally, we have the following result. (4).

Proposition 2: Let consider the coupled system (3)-

- There always exists an Equilibrium without disease, $E_{D F}$, depending on the threshold $\mathcal{N}_{i, m}$ in each patch.

- When $\mathcal{N}_{m}=\left(\mathcal{N}_{i, m}\right)_{i=1, . ., n}>\mathbf{1}_{n}$, then we call DFE, the Disease Free Equilibrium, $\left(S_{h}^{0}, 0,0, A_{m}^{0}, S_{m}^{0}, 0\right)$, where $A_{m}^{0}>0$ and $S_{m}^{0}>0$.

\section{$B$. The basic reproduction number}

Let us now compute a general expression related to an equilibrium without disease. We will consider system (5)-(6), without equation $\frac{d R_{h}}{d t}$, because the human population is constant. The expressions which coming from the other compartments due to the contamination are those in $\frac{d I_{h}}{d t}$ and $\frac{d I_{m}}{d t}$, that is

$$
\left\{\begin{array}{l}
\frac{d I_{h}}{d t}=\operatorname{diag}\left(\beta_{m h}\right) \operatorname{diag}\left(N_{h}\right)^{-1} \operatorname{diag}\left(I_{m}\right) S_{h} \\
\quad-\operatorname{diag}\left(\eta_{h}+\mu_{h}\right) I_{h}+\mathcal{M} \Gamma I_{h}, \\
\frac{d I_{m}}{d t}=\operatorname{diag}\left(\beta_{h m}\right) \operatorname{diag}\left(N_{h}\right)^{-1} \operatorname{diag}\left(I_{h}\right) S_{m} \\
\quad-\operatorname{diag}\left(\mu_{m}\right) I_{m} .
\end{array}\right.
$$

The above equation can be rewritten as follows :

$$
\left\{\begin{array}{l}
\frac{d I_{h}}{d t}=\operatorname{diag}\left(\beta_{m h}\right) \operatorname{diag}\left(N_{h}\right)^{-1} \operatorname{diag}\left(S_{h}\right) I_{m} \\
\quad-\operatorname{diag}\left(\eta_{h}+\mu_{h}\right) I_{h}+\mathcal{M} \Gamma I_{h}, \\
\frac{d I_{m}}{d t}=\operatorname{diag}\left(\beta_{h m}\right) \operatorname{diag}\left(N_{h}\right)^{-1} \operatorname{diag}\left(S_{m}\right) I_{h} \\
-\operatorname{diag}\left(\mu_{m}\right) I_{m},
\end{array}\right.
$$

Here we consider a general equilibrium without disease such that $S_{m} \geq 0$, such that some components could be equal to zero. We compute the Jacobian of the system at a nonnegative equilibrium, without disease, $E_{D F}=$ $\left(A_{m}^{0}, S_{m}^{0}, 0, N_{h}^{0}, 0,0\right)$, which leads to

$$
J_{M}=F-V
$$

where

$F=\left(\begin{array}{cc}0_{n \times n} & \operatorname{diag}\left(\beta_{m h}\right) \\ \operatorname{diag}\left(\beta_{h m}\right) \operatorname{diag}\left(N_{h}^{0}\right)^{-1} \operatorname{diag}\left(S_{m}^{0}\right) & 0_{n \times n}\end{array}\right)$ and

$$
V=\left(\begin{array}{cc}
\left(\eta_{h}+\mu_{h}\right) I d_{n}-\mathcal{M} \Gamma & 0_{n \times n} \\
0_{n \times n} & \operatorname{diag}\left(\mu_{m}\right)
\end{array}\right),
$$

which is invertible. Then, the next generation matrix is:

$$
F V^{-1}=\left(\begin{array}{cc}
0_{n \times n} & f_{12} \\
f_{21} & 0_{n \times n}
\end{array}\right),
$$

where $f_{12}=\operatorname{diag}\left(\beta_{m h}\right)\left(\operatorname{diag}\left(\mu_{m}\right)\right)^{-1}$,

$$
\begin{aligned}
& f_{21}=\operatorname{diag}\left(\beta_{h m}\right) \operatorname{diag}\left(N_{h}^{0}\right)^{-1} \operatorname{diag}\left(S_{m}^{0}\right) \\
& \times\left(\left(\eta_{h}+\mu_{h}\right) I d_{n}-\mathcal{M} \Gamma\right)^{-1} .
\end{aligned}
$$

The basic reproduction number related to $E_{D F}$ is the spectral radius of the next generation matrix, i.e. $\mathcal{R}_{0}^{2}=$ $\rho\left(F V^{-1}\right)$ [33]. After a brief computation, we obtain

$$
\begin{aligned}
& \mathcal{R}_{0}^{2}=\rho\left(\operatorname{diag}\left(\beta_{m h} \beta_{h m}\right)\left(\operatorname{diag}\left(\mu_{m}\right)\right)^{-1} \operatorname{diag}\left(N_{h}^{0}\right)^{-1}\right. \\
& \left.\times \operatorname{diag}\left(S_{m}^{0}\right)\left(\left(\eta_{h}+\mu_{h}\right) I d_{n}-\mathcal{M} \Gamma\right)^{-1}\right) .
\end{aligned}
$$

$\mathcal{R}_{0}^{2}$ is also the general basic reproduction number related to the whole system. Using [33], we have the following result. 
Theorem 3: If $\mathcal{R}_{0}^{2}<1$, then $E_{D F}$ is locally asymptotically stable. If $\mathcal{R}_{0}^{2}>1$, then $E_{D F}$ is unstable.

Remark 2: If $\mathcal{N}_{m}<\mathbf{1}_{n}$, then only the infection free equilibrium (IFE) $E_{0}=\left(S_{h}^{0}, 0,0,0,0,0\right)$ exists and is globally asymptotically stable. If $\mathcal{N}_{m}>\mathbf{1}_{n}$, the IFE still exists but is unstable and the DFE $E^{\#}$ exists. This DFE as we have shown in Theorem 1 is stable. This means that at $\mathcal{N}_{m}=\mathbf{1}_{n}$, we have a transcritical bifurcation.

Remark 3: When $\Gamma=0$, then

$$
\mathcal{R}_{0}^{2}=\max _{i}\left(\mathcal{R}_{0, i}^{2}\right),
$$

where $\mathcal{R}_{0, i}^{2}$ is the basic reproduction number in patch $i$, and, is defined as follows [22]:

$$
\mathcal{R}_{0, i}^{2}=\frac{\beta_{i, m h} \beta_{i, h m}}{\mu_{i, m}\left(\eta_{h}+\mu_{h}\right)} \frac{S_{i, m}^{0}}{N_{i, h}^{0}} .
$$

From the previous computations, we are able to derive interesting results, in particular for the vector control. A first general and obvious result is that when the migration increases, the basic reproduction number also increases. Indeed, $\left(\eta_{h}+\mu_{h}\right) I d_{n}-\mathcal{M} \Gamma$ being an $M-$ matrix, its inverse $\left(\left(\eta_{h}+\mu_{h}\right) I d_{n}-\mathcal{M} \Gamma\right)^{-1}$ is a positive matrix. Moreover

$$
\begin{aligned}
\operatorname{diag}\left(N_{h}^{0}\right)^{-1} \operatorname{diag}\left(S_{m}^{0}\right) & =\left(\begin{array}{cc}
\frac{S_{1, m}^{0}}{N_{1, h}^{0}} & \\
& \ddots \\
& \\
& \frac{S_{n, m}^{0}}{N_{n, h}^{0}}
\end{array}\right) \\
& \leq \max _{1 \leq i \leq n}\left(\frac{S_{i, m}^{0}}{N_{i, h}^{0}}\right) I d_{n} .
\end{aligned}
$$

Thus, we have

$$
\begin{aligned}
& \operatorname{diag}\left(\frac{\beta_{m h} \beta_{h m}}{\mu_{m}}\right) \operatorname{diag}\left(N_{h}^{0}\right)^{-1} \operatorname{diag}\left(S_{m}^{0}\right) \\
& \times\left(\left(\eta_{h}+\mu_{h}\right) I d_{n}-\mathcal{M} \Gamma\right)^{-1} \\
& \leq \max _{1 \leq i \leq n}\left(\frac{\beta_{i, m h} \beta_{i, h m}}{\mu_{i, m}} \frac{S_{i, m}^{0}}{N_{i, h}^{0}}\right)\left(\left(\eta_{h}+\mu_{h}\right) I d_{n}-\mathcal{M} \Gamma\right)^{-1} .
\end{aligned}
$$

Then using a nice property of positive matrices, we have

$$
\begin{aligned}
& \rho\left(\operatorname{diag} \frac{\beta_{m h} \beta_{h m}}{\mu_{m}} \operatorname{diag}\left(N_{h}^{0}\right)^{-1} \operatorname{diag}\left(S_{m}^{0}\right) \times\right. \\
& \left.\times\left(\left(\eta_{h}+\mu_{h}\right) I d_{n}-\mathcal{M} \Gamma\right)^{-1}\right) \\
& \leq \max _{1 \leq i \leq n}\left(\frac{\beta_{i, m h} \beta_{i, h m}}{\mu_{i, m}} \frac{S_{i, m}^{0}}{N_{i, h}^{0}}\right) \rho\left(\left(\left(\eta_{h}+\mu_{h}\right) I d_{n}-\mathcal{M} \Gamma\right)^{-1}\right)
\end{aligned}
$$

But $\left(\eta_{h}+\mu_{h}\right) I d_{n}-\mathcal{M} \Gamma$ being a non singular $M$ matrix, since the stability modulus of $\mathcal{M}$ is $\alpha(\mathcal{M})=0$, where $\alpha(\mathcal{M})=\max \{\operatorname{Re}(\lambda): \lambda$ eigenvalue of $\mathcal{M}\}$, we deduce [9]

$$
\rho\left(\left(\left(\eta_{h}+\mu_{h}\right) I d_{n}-\mathcal{M} \Gamma\right)^{-1}\right)=\frac{1}{\eta_{h}+\mu_{h}},
$$

which implies, using (14), that

$$
\mathcal{R}_{0}^{2} \leq \max _{i}\left(\mathcal{R}_{0, i}^{2}\right) .
$$

Using the same reasoning it is possible to show that

$$
\min _{i}\left(\mathcal{R}_{0, i}^{2}\right) \leq \mathcal{R}_{0}^{2} .
$$

Altogether, we summarize in the following proposition

Proposition 3: The Basic reproduction Number of the patch system verifies

$$
\min _{i}\left(\mathcal{R}_{0, i}^{2}\right) \leq \mathcal{R}_{0}^{2} \leq \max _{i}\left(\mathcal{R}_{0, i}^{2}\right) .
$$

Thus, human movements can induce a spreading of the epidemiological risk in places where local basic reproduction numbers are low, when some places have large local reproduction number, i.e. greater than 1 .

Let us now consider a particular case of $n$ patches with the same population $N_{i, h}^{0}=N_{1, h}$, the same capacity $K_{i}=K_{1}$, and the same parameters values in each patch, for $i=2, \ldots, n$, such that $\mathcal{N}_{m}>1$. Then we have the same equilibrium for the susceptible mosquito population, i.e. $S_{i, m}^{0}=S_{1, m}^{0}$, for $i=2, \ldots, n$. Thus the basic reproduction number reduces to

$$
\begin{aligned}
& \mathcal{R}_{0}^{2}=\rho\left(\operatorname{diag}\left(\frac{\beta_{m h} \beta_{h m}}{\mu_{m}}\right) \operatorname{diag}\left(N_{h}^{0}\right)^{-1} \operatorname{diag}\left(S_{m}^{0}\right)\right. \\
& \left.\left(\left(\eta_{h}+\mu_{h}\right) I d_{n}-\mathcal{M}\right)^{-1} \Gamma\right)=\frac{\beta_{m h} \beta_{h m}}{\mu_{m}} \frac{S_{1, m}^{0}}{N_{1, h}^{0}} \\
& \times \rho\left(\left(\left(\eta_{h}+\mu_{h}\right) I d_{n}-\mathcal{M} \Gamma\right)^{-1}\right),
\end{aligned}
$$

with

$$
\rho\left(\left(\left(\eta_{h}+\mu_{h}\right) I d_{n}-\mathcal{M} \Gamma\right)^{-1}\right)=\frac{1}{\eta_{h}+\mu_{h}}
$$

which implies that

$$
\mathcal{R}_{0}^{2}=\mathcal{R}_{0,1}^{2}=\mathcal{R}_{0, i}^{2}=\frac{\beta_{1, h m} \beta_{1, m h}}{\mu_{1, m}\left(\eta_{h}+\mu_{h}\right)} \frac{S_{1, m}^{0}}{N_{1, h}^{0}} .
$$

In this particular case, human movements has no impact on the basic reproduction number. Thus, for cities of equal size, and with the same biological parameters whatever the migration, the global and local risks are the same. This unexpected result is due to the fact that $\left(\left(\eta_{h}+\mu_{h}\right) I d_{n}-\mathcal{M} \Gamma\right)^{-1}$ has always the same spectral radius, $\frac{1}{\left(\eta_{h}+\mu_{h}\right)}$, whatever the matrices $\Gamma$ and $\mathcal{M}$. 
Let us now consider a two-patches example, with $\mathcal{N}_{1, m}>1$ and $\mathcal{N}_{m, 2}<1$. Thus, after straightforward computations, the basic reproduction number becomes

$\mathcal{R}_{0}^{2}=\frac{\beta_{1, h m} \beta_{1, m h}}{\mu_{m}\left(\eta_{h}+\mu_{h}\right)} \frac{\left(\eta_{h}+\mu_{h}+\gamma_{1} m_{12}\right)}{\left(\eta_{h}+\mu_{h}+\gamma_{1}\left(m_{12}+m_{21}\right)\right)} \frac{S_{1, m}^{0}}{N_{1, h}^{0}}$,

which leads to

$$
\mathcal{R}_{0}^{2}=\mathcal{R}_{0,1}^{2}\left(1-\gamma_{1} \frac{m_{21}}{\left(\eta_{h}+\mu_{h}+\gamma_{1}\left(m_{21}+m_{12}\right)\right)}\right)
$$

indicating that if the infected population is in one patch, with infectious mosquitoes, has back and forth movement with another patch which is free of mosquitoes, then the basic reproduction ratio will decrease....In particular if all infected people go out from the infected area, this will lower the epidemiological risk....

\section{Global asymptotic stability of the DFE}

In this section, we study the global asymptotic stability (GAS) of the DFE of coupled system (5)-(6). We assume that the population in each patch is constant, i.e. $N_{i, h}=$ $N_{i, h}^{0}$. Set

$$
\begin{aligned}
& \mathcal{R}_{G A S}^{2}=\rho\left(\operatorname{diag}\left(\beta_{m h} \beta_{h m}\right)\left(\operatorname{diag}\left(\mu_{m}\right)\right)^{-1} \operatorname{diag}\left(N_{h}^{0}\right)^{-1}\right. \\
& \left.\times \operatorname{diag}\left(S_{\max }\right)\left(\left(\eta_{h}+\mu_{h}\right) I d_{n}-\mathcal{M} \Gamma\right)^{-1}\right),
\end{aligned}
$$

with

$$
S_{\max }=\max \left(S_{m}(0), S_{m}^{0}\right) .
$$

We have the following result

Theorem 4: : The DFE of the coupled system (5)(6) is globally asymptotically stable in the nonnegative orthant, if $\mathcal{R}_{G A S}^{2}<1$.

Proof: Let us consider Eqs. $(5)_{3}$ and $(6)_{2}$. Using the fact that $S_{h}$ is bounded, i.e. $S_{h} \leq N_{h}^{0}$, and $S_{m}<S_{\max }$, we obtain the following linear differential inequations system:

$$
\left(\begin{array}{c}
\frac{d I_{m}}{d t} \\
\frac{d I_{h}}{d t}
\end{array}\right) \leq\left(\begin{array}{cc}
-\operatorname{diag}\left(\mu_{m}\right) & g_{21} \\
\operatorname{diag}\left(\beta_{m h}\right) & -g_{22}
\end{array}\right)\left(\begin{array}{c}
I_{m} \\
I_{h}
\end{array}\right),
$$

where $g_{21}=\operatorname{diag}\left(\beta_{h m}\right) \operatorname{diag}\left(N_{h}^{0}\right)^{-1} \operatorname{diag}\left(S_{\max }\right)$, $g_{22}=\left(\operatorname{diag}\left(\eta_{h}+\mu_{h}\right)-\mathcal{M} \Gamma\right)$. Let

$$
G=\left(\begin{array}{cc}
-\operatorname{diag}\left(\mu_{m}\right) & g_{21} \\
\operatorname{diag}\left(\beta_{m h}\right) & -g_{22}
\end{array}\right) .
$$

Note that $G$ is a Metzler matrix, which admits a regular splitting [9] (close similar to the regular splitting obtained to compute the basic reproduction number $\mathcal{R}_{0}^{2}$ ), $N+M$, with

$$
M=\left(\begin{array}{cc}
0 & \operatorname{diag}\left(\beta_{h m}\right) \operatorname{diag}\left(N_{h}^{0}\right)^{-1} \operatorname{diag}\left(S_{\max }\right) \\
\operatorname{diag}\left(\beta_{m h}\right) & 0
\end{array}\right)
$$

and

$$
N=\left(\begin{array}{cc}
-\operatorname{diag}\left(\mu_{m}\right) & 0 \\
0 & -\left(\operatorname{diag}\left(\eta_{h}+\mu_{h}\right)-\mathcal{M} \Gamma\right)
\end{array}\right)
$$

Thus, using [9], $G$ is Metzler stable if $\rho\left(-N^{-1} M\right)<1$. A simple computation gives

$$
-N^{-1} M=\left(\begin{array}{cc}
0 & n m_{12} \\
n m_{21} & 0
\end{array}\right),
$$

where

$$
\begin{aligned}
& n m_{12}=\operatorname{diag}\left(\beta_{h m}\right) \operatorname{diag}\left(N_{h}^{0}\right)^{-1} \operatorname{diag}\left(S_{\max }\right) \\
& \times\left(\operatorname{diag}\left(\mu_{m}\right)\right)^{-1},
\end{aligned}
$$

$n m_{21}=\left(\operatorname{diag}\left(\eta_{h}+\mu_{h}\right)-\mathcal{M} \Gamma\right)^{-1} \operatorname{diag}\left(\beta_{m h}\right)$. Then, $\rho\left(-N^{-1} M\right)<1$ if and only if

$$
\begin{aligned}
& \mathcal{R}_{G A S}^{2}=\rho\left(\operatorname{diag}\left(\frac{\beta_{m h} \beta_{h m}}{\mu_{m}}\right) \operatorname{diag}\left(N_{h}^{0}\right)^{-1}\right. \\
& \times \operatorname{diag}\left(S_{\max }\right)\left(\left(\eta_{h}+\mu_{h}\right) I d_{n}-\mathcal{M} \Gamma\right)^{-1}<1 .
\end{aligned}
$$

Thus, using a comparison principle [26], we have

$$
\lim _{t \rightarrow+\infty} I_{h}=\lim _{t \rightarrow+\infty} I_{m}=0 .
$$

Then, having $-\left(\mu_{h} I d_{n}-\mathcal{M}\right) \leq 0$, we deduce that $\lim _{t \rightarrow+\infty} R_{h}=0$. Since the total population in each patch is constant, and using the fact that $S_{h}+I_{h}+R_{h}=N_{h}$, we deduce that $\lim _{t \rightarrow+\infty} S_{h}=N_{h}$.

Obviously we have $\mathcal{R}_{0}^{2} \leq \mathcal{R}_{G A S}^{2}$. Thus since $\mathcal{R}_{G A S}^{2}<$ 1 , we have uniqueness of the $E_{D F}$, which implies that $\lim _{t \rightarrow+\infty} A_{m}=A_{m}^{0}$, and $\lim _{t \rightarrow+\infty} S_{m}=S_{m}^{0}$. Then, one can conclude that the $E_{D F}$ is GAS when $\mathcal{R}_{G A S}^{2}<1$. This achieves the proof. $\mathcal{R}_{0}^{2}$.

Remark 4: :When $S_{\max }=S_{m}^{0}$, we have $\mathcal{R}_{G A S}^{2}=$

Remark 5: : Our result generalized to a metapopulation the results obtained in [18], [19], [22]. 


\section{SpREADING OF THE DISEASE}

First of all, when $\mathcal{M} \neq 0$, we show with a simple example that having an endemic equilibrium in some patches and a Disease Free equilibrium in other patches, is impossible.

Indeed, we consider a two-patches system assuming that in the first patch $\mathcal{R}_{0,1}<1$ and in the second ones, $\mathcal{R}_{0,2}>1$. Looking for an equilibrium leads to the following systems to solve

$$
\begin{aligned}
& \text { (Patch 1) }\left\{\begin{array}{l}
\mu_{h} N_{1, h}-\beta_{1, m h} \frac{I_{1, m}}{N_{1, h}} S_{1, h} \\
-\mu_{h} S_{1, h}+m_{12}^{S} S_{2, h}-m_{21}^{S} S_{1, h}=0, \\
\beta_{1, m h} \frac{I_{1, m}}{N_{1, h}} S_{1, h}-\left(\mu_{h}+\eta_{h}\right) I_{1, h} \\
+\gamma_{2} m_{12}^{I} I_{2, h}-\gamma_{1} m_{21}^{I} I_{1, h}=0,
\end{array}\right. \\
& \text { (Patch 2) }\left\{\begin{array}{l}
\mu_{h} N_{2, h}-\beta_{2, m h} \frac{I_{2, m}}{N_{2, h}} S_{2, h}-\mu_{h} S_{2, h} \\
+m_{21}^{S} S_{1, h}-m_{12}^{S} S_{2, h}=0, \\
\beta_{2, m h} \frac{I_{2, m}}{N_{2, h}} S_{2, h}-\left(\mu_{h}+\eta_{h}\right) I_{2, h} \\
+\gamma_{1} m_{21}^{I} I_{1, h}-\gamma_{2} m_{12}^{I} I_{2, h}=0 .
\end{array}\right.
\end{aligned}
$$

From $(\text { Patch } 1)_{2}$, we deduce

$\gamma_{2} m_{12}^{I} I_{2, h}=\left(\mu_{h}+\eta_{h}+\gamma_{1} m_{21}^{I}\right) I_{1, h}-\beta_{1, m h} \frac{I_{1, m}}{N_{1, h}} S_{1, h}$.

We assume $\gamma_{1}>0$. Since $\mathcal{R}_{0,1}<1$ in the first patch, then one possible equilibrium verifies $I_{1, h}=I_{1, m}=0$. Using $(15)_{2}$, we have immediately $I_{2, h}=0$ in the second patch. Then using the fact that

$$
\beta_{2, h m} \frac{I_{2, h}}{N_{2, h}} S_{2, m}=\mu_{2, m} I_{2, m},
$$

we deduce that $I_{2, m}=0$. Thus, assuming movements in the infectious population implies automatically that we cannot have co-existence of an endemic equilibrium in one patch and a disease free equilibrium in the other one. Therefore, an endemic equilibrium for the patchy system is an equilibrium without zero component.

Existence of an endemic equilibrium is not obvious to show in a metapopulation model. However, it is possible to have some insights in the dynamic of the disease, in particular regarding its spreading. Our preliminary results allows us to conjecture that a unique endemic equilibrium exists. Let us now stated this result for $n$ patches:

Proposition 4: Consider system (5)-(6).

1) When $\mathcal{M}=0$, a unique endemic equilibrium exists, if $\mathcal{R}_{0, i}^{2}>1$ in each patch $i$.
2) When $\mathcal{M} \neq 0$, the only equilibrium with one zero component is $E_{D F}$.

Proof: Let us first show the existence of a possible equilibrium. Then, using some of the computations derived in [19], we can derive explicit solution for the endemic equilibrium.... Let us consider system (3)-(4) or system (5)-(6) at the endemic equilibrium $\left(S_{h}^{*}, I_{h}^{*}, R_{h}^{*}, A_{m}^{*}, S_{m}^{*}, I_{m}^{*}\right)$ with $I_{h}^{*} \neq 0$ and $I_{m}^{*} \neq 0$ in each patch $i$. First, using system (6), and setting $\frac{d S_{h}}{d t}(t)=\frac{d I_{h}}{d t}(t)=\frac{d R_{h}}{d t}(t)=0$, we obtain the following relations:

$$
\left\{\begin{array}{l}
\left(\operatorname{diag}\left(\mu_{h}\right)-\mathcal{M}\right) R_{h}^{*}=\eta_{h} I_{h}^{*}, \\
\left(\left(\operatorname{diag}\left(N_{h}^{*}\right)\right)^{-1} \operatorname{diag}\left(\beta_{m h} I_{m}^{*}\right)-\mathcal{M}\right) S_{h}^{*} \\
\quad=\mu_{h}\left(I_{h}^{*}+R_{h}^{*}\right), \\
\left(\operatorname{diag}\left(\eta_{h}+\mu_{h}\right)-\mathcal{M} \Gamma\right) I_{h}^{*}=\left(\operatorname{diag}\left(N_{h}^{*}\right)\right)^{-1} \\
\times \operatorname{diag}\left(\beta_{m h} I_{m}^{*}\right) S_{h}^{*} .
\end{array}\right.
$$

1) From Eq. $(16)_{2}$, we deduce

$\left(\left(\operatorname{diag}\left(N_{h}^{*}\right)\right)^{-1} \operatorname{diag}\left(\beta_{m h} I_{m}^{*}\right)+\mu_{h} I d_{n}-\mathcal{M}\right) S_{h}^{*}=\mu_{h} N_{h}^{*}$,

Thus using $(16)_{2}$ and (17), we deduce

$$
\begin{aligned}
& \left(\operatorname{diag}\left(\eta_{h}+\mu_{h}\right)-\mathcal{M} \Gamma\right) I_{h}^{*}=\mu_{h} \operatorname{diag}\left(\frac{\beta_{m h} I_{m}^{*}}{N_{h}^{*}}\right) \\
& \times\left(\left(\operatorname{diag}\left(N_{h}^{*}\right)\right)^{-1} \operatorname{diag}\left(\beta_{m h} I_{m}^{*}\right)+\mu_{h} I d_{n}-\mathcal{M}\right)^{-1} N_{h}^{*},
\end{aligned}
$$

that is

$$
\begin{aligned}
& I_{h}^{*}=\mu_{h}\left(\operatorname{diag}\left(\eta_{h}+\mu_{h}\right)-\mathcal{M} \Gamma\right)^{-1} \operatorname{diag}\left(\frac{\beta_{m h} I_{m}^{*}}{N_{h}^{*}}\right) \\
& \times\left(\left(\operatorname{diag}\left(N_{h}^{*}\right)\right)^{-1} \operatorname{diag}\left(\beta_{m h} I_{m}^{*}\right)+\mu_{h} I d_{n}-\mathcal{M}\right)^{-1} N_{h}^{*} .
\end{aligned}
$$

Now, we have two cases to consider: $\mathcal{M}=0$ and $\mathcal{M} \neq$ 0 .

Suppose $\mathcal{M}=0$. Then the patches are disjoints and in this case it suffices to follow the computations given in [19], [18]. Indeed, we have

$$
I_{i, m}^{*}=\frac{\eta_{i, A} \beta_{i, h m} I_{i, h}^{*}}{\mu_{i, a m}\left(\mu_{i, m} N_{i, h}^{*}+\beta_{i, h m} I_{i, h}^{*}\right)}\left(1-\frac{1}{\mathcal{N}_{i, m}}\right) \alpha_{i} K_{i},
$$

and from Eq. 16$)_{3}$, we have

$$
\frac{I_{i, h}^{*}}{N_{i, h}^{*}}=\frac{\mu_{h}}{\eta_{h}+\mu_{h}} \frac{\beta_{i, m h} I_{i, m}^{*}}{\beta_{i, m h} I_{i, m}^{*}+\mu_{h} N_{i, h}^{*}} .
$$

From the two previous equation, we deduce:

$$
I_{i, m}^{*}=\frac{\mu_{h} \mu_{i, m}}{\beta_{i, m h}\left(1+\frac{\mu_{h}}{\eta_{h}+\mu_{h}} \beta_{i, h m}\right)}\left(\mathcal{R}_{0, i}^{2}-1\right) N_{i, h}^{*} .
$$


Note that Eq. (21) has a sense if and only if $\mathcal{R}_{0, i}^{2}>1$, where $\mathcal{R}_{0, i}^{2}$ is defined as in Eq. (14). Thus each patch admits an endemic equilibrium if $\mathcal{R}_{0, i}^{2}>1$.

2) Using some of the previous computations, we now suppose that $\mathcal{M} \neq 0$. In that case, the computations are absolutely not obvious and we need to show the existence of the endemic equilibrium, using fixed point argument. We will use Theorem 2.1 given in [24]. Using the previous computations we have to combine Eqs. (18) and (19), which lead to a fixed point problem, $G(X)=X$, with

$$
\begin{aligned}
& G(X)=\mu_{h}\left(\operatorname{diag}\left(\eta_{h}+\mu_{h}\right)-\mathcal{M} \Gamma\right)^{-1} \\
& \times \operatorname{diag}\left(\frac{\beta_{i, m h}}{N_{i, h}^{*}} u_{i, h m}\left(1-\frac{1}{\mathcal{N}_{i, m}}\right) \alpha_{i} K_{i}\right) \\
& \times\left(u^{*}+\mu_{h} I d_{n}-\mathcal{M}\right)^{-1} N_{h}^{*},
\end{aligned}
$$

where $u_{i, h m}=\frac{\eta_{i, A} \beta_{i, h m} X_{i, h}}{\mu_{i, m}\left(\mu_{i, m} N_{i, h}^{*}+\beta_{i, h m} X_{i, h}\right)}$ and $u^{*}=$ $\left(\operatorname{diag}\left(N_{h}^{*}\right)\right)^{-1} \operatorname{diag}\left(\beta_{m h} u_{i, h m}\left(1-\frac{1}{\mathcal{N}_{i, m}}\right) \alpha_{i} K_{i}\right)$.

Note carefully that the matrices

$$
\begin{aligned}
& \left(\operatorname{diag}\left(N_{h}^{*}\right)\right)^{-1} \operatorname{diag}\left(\beta_{i, m h} u_{i, h m}\left(1-\frac{1}{\mathcal{N}_{i, m}}\right) \alpha_{i} K_{i}\right) \\
& +\mu_{h} I d_{n}-\mathcal{M},
\end{aligned}
$$

and

$$
\operatorname{diag}\left(\eta_{h}+\mu_{h}\right)-\mathcal{M} \Gamma,
$$

are M-Matrices and thus their inverses are positive.

We now verify the assumptions of Theorem 2.1 in [24]: it is obvious that $G$ is continuous, monotone nondecreasing, and strictly sublinear. $G$ is bounded and stay in the nonnegative orthant. Moreover $G(0)=0$, and $G^{\prime}(0)$ exists and is irreducible. Thus from Theorem 2.1 [24], we can deduce that the only equilibrium with one zero component is the $E_{D F}$. It means that if at least one patch is infected, then all patches will become infected through population movements, unless $\Gamma=0$ or $\mathcal{M}=0$.

Remark 6: From the epidemiological point of view, the previous result states that if at least one patch is infected, then every patch will become infected, due to Human movements when $\gamma>0$. This result seems obvious, but it clearly shows that if early vector control is not undertaken in places where infective people are recorded the disease will spread due to Human movements... and, of course, it will far more difficult to control the epidemic.

Remark 7: In fact, using the same theorem we could go further, if we were able to show that $\rho\left(G^{\prime}(0)\right)>1$, then we could deduce the existence of a unique positive fixed point. In our case, we have

$$
\begin{aligned}
& G^{\prime}(0)=\operatorname{diag}\left(\eta_{h}+\mu_{h}\right)\left(\operatorname{diag}\left(\eta_{h}+\mu_{h}\right)-\mathcal{M} \Gamma\right)^{-1} \\
& \times \operatorname{diag}\left(\frac{1}{\left(\eta_{h}+\mu_{h}\right)} \frac{\eta_{i, A} \beta_{i, m h} \beta_{i, h m}}{\mu_{i, m} \mu_{i, m} N_{i, h}^{*}}\left(1-\frac{1}{\mathcal{N}_{i, m}}\right) \alpha_{i} K_{i}\right) \\
& \times \operatorname{diag}\left(\mu_{h}\right)\left(\mu_{h} I d_{n}-\mathcal{M}\right)^{-1} .
\end{aligned}
$$

In the diagonal matrix, we recognize the local basic reproduction Number $R_{i, 0}^{2}$. Thus

$$
\begin{aligned}
& G^{\prime}(0)=\operatorname{diag}\left(\eta_{h}+\mu_{h}\right)\left(\operatorname{diag}\left(\eta_{h}+\mu_{h}\right)-\mathcal{M} \Gamma\right)^{-1} \\
& \times \operatorname{diag}\left(\mathcal{R}_{i, 0}^{2}\right) \operatorname{diag}\left(\mu_{h}\right)\left(\mu_{h} I d_{n}-\mathcal{M}\right)^{-1} .
\end{aligned}
$$

Although in the previous equality, all "ingredients" are there. In particular, we have

$$
\begin{aligned}
\rho\left(\left(\operatorname{diag}\left(\eta_{h}+\mu_{h}\right)-\mathcal{M} \Gamma\right)^{-1}\right) & =\operatorname{diag}\left(\frac{1}{\left(\eta_{h}+\mu_{h}\right)}\right), \\
\rho\left(\left(\operatorname{diag}\left(\mu_{h}\right)-\mathcal{M}\right)^{-1}\right) & =\operatorname{diag}\left(\frac{1}{\mu_{h}}\right) .
\end{aligned}
$$

Therefore, we can only conjecture that

$$
\rho\left(G^{\prime}(0)\right) \geq \max _{i} \mathcal{R}_{i, 0}^{2} .
$$

Then, $\rho\left(G^{\prime}(0)\right)>1$ if and only if there exists at least one patch $k$ where $\mathcal{R}_{k, 0}^{2}>1$.

Remark 8: When $\mathcal{M} \neq 0$, we conjecture also that there exists a unique endemic equilibrium when $\max _{i} R_{0, i}^{2}>1$. This result is particularly useful: it shows that local and fast intervention when Chikungunya cases are suspected is the best way to stop the spreading of the disease. Fast and localized intervention is now the standard procedure in Réunion Island.

\section{AppliCATiOnS}

We consider a two patch-model in order to illustrate the complexity of the results depending on the population size in each patch and the movement rates between patches. In particular, since the choices for the migration matrix are numerous, we would like to emphasize the fact that the construction of the migration model is highly sensitive. From these choices, the results may change drastically and then drive to wrong decisions either to decide or not about field interventions in order to control a disease or not.

The main advantage of this approach with two patches is that we are able to provide an analytical formula for $\mathcal{R}_{0}^{2}$ and thus to discuss the impacts of different parameters. For simplification, we assume that $\mu_{m}=\mu_{a m}$. 
Then, Equations (18) and (19) are equivalent to the Moreover following fixed point problem

$$
\left(\begin{array}{c}
X \\
Y
\end{array}\right)=\left(\begin{array}{c}
F(Y) \\
G(X)
\end{array}\right)
$$

with

$$
\begin{aligned}
& F(Y)=\mu_{h}\left(\operatorname{diag}\left(\eta_{h}+\mu_{h}\right)-\mathcal{M} \Gamma\right)^{-1} \operatorname{diag}\left(\frac{\beta_{m h} Y}{N_{h}^{*}}\right) \\
& \times\left(\operatorname{diag}\left(\frac{\beta_{m h} Y}{N_{h}^{*}}\right)+\mu_{h} I d_{2}-\mathcal{M}\right)^{-1} N_{h}^{*}, \\
& G_{i}(X)=\frac{\eta_{i, A} \beta_{i, h m} X_{i}}{\mu_{i, m}\left(\mu_{i, m} N_{i, h}^{*}+\beta_{i, h m} X_{i}\right)}\left(1-\frac{1}{\mathcal{N}_{i, m}}\right) \alpha_{i} K_{i} \\
& \begin{array}{l}
\overline{\left(\frac{\beta_{1, m h} Y_{1}}{N_{1, h}^{*}}+\mu_{h}+m_{21}\right)\left(\frac{\beta_{2, m h} Y_{2}}{N_{2, h}^{*}}+\mu_{h}+m_{12}\right)-m_{21} m_{12}} \\
\times\left(\frac{\mu_{h}}{\left(\eta_{h}+\mu_{h}\right)\left(\eta_{h}+\mu_{h}+\gamma_{1} m_{21}+\gamma_{2} m_{12}\right)}\right) \times
\end{array}
\end{aligned}
$$

$$
\begin{aligned}
& \mu_{h}\left(\operatorname{diag}\left(\eta_{h}+\mu_{h}\right)-\mathcal{M} \Gamma\right)^{-1} \\
& =\frac{\mu_{h}}{\left(\eta_{h}+\mu_{h}\right)\left(\eta_{h}+\mu_{h}+\gamma_{1} m_{21}+\gamma_{2} m_{12}\right)} \\
& \times\left(\begin{array}{cc}
\eta_{h}+\mu_{h}+\gamma_{2} m_{12} & \gamma_{2} m_{12} \\
\gamma_{1} m_{21} & \eta_{h}+\mu_{h}+\gamma_{1} m_{21}
\end{array}\right) .
\end{aligned}
$$

Finally $F_{\gamma}(Y)=$

Let us compute $G^{\prime}(0)$ for a two patches system with a human population $\left(N_{1, h}, N_{2, h}\right)$. The movement matrix is chosen such that $\mathcal{M} N_{h}^{*}=0$. For instance, $\mathcal{M}$ could be chosen as follows:

$$
\mathcal{M}=\left(\begin{array}{cc}
-1 & \frac{N_{1, h}^{*}}{N_{2, h}^{*}} \\
1 & -\frac{N_{1, h}^{*}}{N_{2, h}^{*}}
\end{array}\right)
$$

In particular, we deduce that $\operatorname{diag}(\alpha)-\mathcal{M}$ is an $M$ matrix when at least one $\alpha_{i}>0$, which implies that

$$
\left(\left(\begin{array}{cc}
\alpha_{1} & 0 \\
0 & \alpha_{2}
\end{array}\right)-\mathcal{M}\right)^{-1}=
$$

$\frac{1}{\left(\alpha_{1}+m_{21}\right)\left(\alpha_{2}+m_{12}\right)-m_{12} m_{21}}\left(\begin{array}{cc}\alpha_{2}+m_{12} & m_{12} \\ m_{21} & \alpha_{1}+m_{21}\end{array}\right)>0$.

Using the previous result, we compute

$$
\begin{aligned}
& \left(\operatorname{diag}\left(\frac{\beta_{m h} Y}{N_{h}^{*}}\right)+\mu_{h} I d_{2}-\mathcal{M}\right)^{-1} \\
& =\frac{1}{\left(\frac{\beta_{1, m h} Y_{1}}{N_{1, h}^{*}}+\mu_{h}+m_{21}\right)\left(\frac{\beta_{2, m h} Y_{2}}{N_{2, h}^{*}}+\mu_{h}+m_{12}\right)-m_{21}} \\
& *\left(\begin{array}{cc}
\frac{\beta_{2, m h} Y_{2}}{N_{2, h}^{*}}+\mu_{h}+m_{12} & m_{12} \\
m_{21} & \frac{\beta_{1, m h} Y_{1}}{N_{1, h}^{*}}+\mu_{h}+m_{21}
\end{array}\right)
\end{aligned}
$$

and

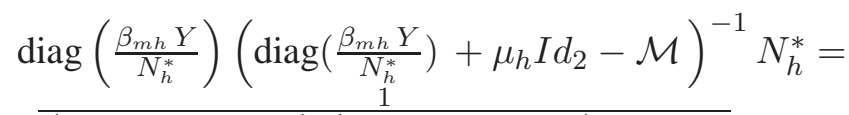

$$
\begin{aligned}
& \overline{\left(\frac{\beta_{1, m h} Y_{1}}{N_{1, h}^{*}}+\mu_{h}+m_{21}\right)\left(\frac{\beta_{2, m h} Y_{2}}{N_{2, h}^{*}}+\mu_{h}+m_{12}\right)-m_{21} m_{12}} \\
& *\left(\begin{array}{c}
\beta_{1, m h} Y_{1}\left(\frac{\beta_{2, m h} Y_{2}}{N_{2, h}^{*}}+\mu_{h}+m_{12}+m_{12} \frac{N_{2, h}^{*}}{N_{1, h}^{*}}\right. \\
\beta_{2, m h} Y_{2}\left(\frac{\beta_{1, m h} Y_{1}}{N_{1, h}^{*}}+\mu_{h}+m_{21}+m_{21} \frac{N_{1, h}^{*}}{N_{2, h}^{*}}\right.
\end{array}\right) . \\
& F_{\gamma}^{\prime}(0)=\frac{1}{\Delta_{1}}\left(\begin{array}{cc}
F_{\gamma}^{\prime}(11) & F_{\gamma}^{\prime}(12) \\
F_{\gamma}^{\prime}(21) & F_{\gamma}^{\prime}(22)
\end{array}\right), \text { where } \\
& \Delta_{1}=\left(\eta_{h}+\mu_{h}\right)\left(\eta_{h}+\mu_{h}+\gamma_{1} m_{21}+\gamma_{2} m_{12}\right) \text {, } \\
& F_{\gamma}^{\prime}(11)=\left(\eta_{h}+\mu_{h}+\gamma_{2} m_{12}\right) \beta_{1, m h} \frac{\mu_{h}+m_{12}+m_{12} \frac{N_{2, h}^{*}}{N_{1, h}^{*}}}{\mu_{h}+m_{12}+m_{21}},
\end{aligned}
$$$$
\left(\begin{array}{cc}
\eta_{h}+\mu_{h}+\gamma_{2} m_{12} & \gamma_{2} m_{12} \\
\gamma_{1} m_{21} & \eta_{h}+\mu_{h}+\gamma_{1} m_{21}
\end{array}\right) \times
$$$$
\left(\begin{array}{c}
\beta_{1, m h} Y_{1}\left(\frac{\beta_{2, m h} Y_{2}}{N_{2, h}^{*}}+\mu_{h}+m_{12}+m_{12} \frac{N_{2, h}^{*}}{N_{1, h}^{*}}\right) \\
\beta_{2, m h} Y_{2}\left(\frac{\beta_{1, m h} Y_{1}}{N_{1, h}^{*}}+\mu_{h}+m_{21}+m_{21} \frac{N_{1, h}^{*}}{N_{2, h}^{*}}\right)
\end{array}\right)
$$$$
=\frac{1}{\left(\frac{\beta_{1, m h} Y_{1}}{N_{1, h}^{*}}+\mu_{h}+m_{21}\right)\left(\frac{\beta_{2, m h} Y_{2}}{N_{2, h}^{*}}+\mu_{h}+m_{12}\right)-m_{21} m_{12}}
$$$$
\frac{\mu_{h}}{\left(\eta_{h}+\mu_{h}\right)\left(\eta_{h}+\mu_{h}+\gamma_{1} m_{21}+\gamma_{2} m_{12}\right)} \times
$$$$
\left(\begin{array}{c}
\left(\eta_{h}+\mu_{h}+\gamma_{2} m_{12}\right) w_{1, m h}+\gamma_{2} m_{12} w_{2, m h} \\
\gamma_{1} m_{21} w_{1, m h}+\left(\eta_{h}+\mu_{h}+\gamma_{1} m_{21}\right) w_{2, m h}
\end{array}\right),
$$

where

$$
\begin{aligned}
& w_{1, m h}=\beta_{1, m h} Y_{1}\left(\frac{\beta_{2, m h} Y_{2}}{N_{2, h}^{*}}+\mu_{h}+m_{12}+m_{12} \frac{N_{2, h}^{*}}{N_{1, h}^{*}}\right), \\
& w_{2, m h}=\beta_{2, m h} Y_{2}\left(\frac{\beta_{1, m h} Y_{1}}{N_{1, h}^{*}}+\mu_{h}+m_{21}+m_{21} \frac{N_{1, h}^{*}}{N_{2, h}^{*}}\right) .
\end{aligned}
$$

Then

$$
\begin{aligned}
& F_{\gamma}(Y)=\frac{\mu_{h}}{\left(\eta_{h}+\mu_{h}\right)\left(\eta_{h}+\mu_{h}+\gamma_{1} m_{21}+\gamma_{2} m_{12}\right)} \times \\
& \overline{\left(\frac{\beta_{1, m h} Y_{1}}{N_{1, h}^{*}}+\mu_{h}+m_{12}\right)\left(\frac{\beta_{2, m h} Y_{2}}{N_{2, h}^{*}}+\mu_{h}+m_{21}\right)-m_{21} m_{12}} \\
& \left(\begin{array}{c}
\left(\eta_{h}+\mu_{h}+\gamma_{2} m_{12}\right) w_{1, m h}+\gamma_{2} m_{12} w_{2, m h} \\
\gamma_{1} m_{21} w_{1, m h}+\left(\eta_{h}+\mu_{h}+\gamma_{1} m_{21}\right) w_{2, m h}
\end{array}\right)
\end{aligned}
$$


$S$ Bowong at al., A patchy model for Chikungunya-like diseases

$$
\begin{aligned}
& F_{\gamma}^{\prime}(12)=\gamma_{2} m_{12} \beta_{2, m h} \frac{\mu_{h}+m_{21}+m_{21} \frac{N_{1, h}^{*}}{N_{2, h}^{*}}}{\mu_{h}+m_{12}+m_{21}}, \\
& F_{\gamma}^{\prime}(21)=\gamma_{1} m_{21} \beta_{1, m h} \frac{\mu_{h}+m_{12}+m_{12} \frac{N_{2, h}^{*}}{N_{1, h}^{*}}}{\mu_{h}+m_{12}+m_{21}},
\end{aligned}
$$

The characteristic polynomial gives

$F_{\gamma}^{\prime}(22)=\left(\eta_{h}+\mu_{h}+\gamma_{1} m_{21}\right) \beta_{2, m h} \frac{\mu_{h}+m_{21}+m_{21} \frac{N_{1, h}^{*}}{N_{2, h}}}{\mu_{h}+m_{12}+m_{21}} .+\left(\frac{1}{\eta_{h}+\mu_{h}+\gamma_{1} m_{21}+\gamma_{2} m_{12}}\right)^{2}\left(\left(\eta_{h}+\mu_{h}+\gamma_{2} m_{12}\right)\right.$

Then using the fact that

$$
m_{21} N_{1, h}^{*}=m_{12} N_{2, h}^{*},
$$

we deduce

$$
F_{\gamma}^{\prime}(0)=\frac{1}{\Delta_{1}}\left(\begin{array}{cc}
f_{\gamma}(11) & \gamma_{2} m_{12} \beta_{2, m h} \\
\gamma_{1} m_{21} \beta_{1, m h} & f_{\gamma}(22)
\end{array}\right),
$$

where $f_{\gamma}(11)=\left(\eta_{h}+\mu_{h}+\gamma_{2} m_{12}\right) \beta_{1, m h}, f_{\gamma}(22)=$ $\left(\eta_{h}+\mu_{h}+\gamma_{1} m_{21}\right) \beta_{2, m h}$.

Finally, with

$$
G^{\prime}(0)=\left(\begin{array}{cc}
g_{1, h m}^{\prime} & 0 \\
0 & g_{2, h m}^{\prime}
\end{array}\right)
$$

where

$$
\begin{aligned}
g_{1, h m}^{\prime} & =\frac{\eta_{1, A} \beta_{1, h m}}{\mu_{1, m} \mu_{1, m} N_{1, h}^{*}}\left(1-\frac{1}{\mathcal{N}_{1}}\right) \alpha_{1} K_{1} \\
& =\frac{\beta_{1, h m}}{\mu_{1, m}} \frac{S_{m, 1}^{0}}{N_{1, h}^{0}} \\
g_{2, h m}^{\prime} & =\frac{\eta_{2, A} \beta_{2, h m}}{\mu_{2, m a} \mu_{2, m} N_{2, h}^{*}}\left(1-\frac{1}{\mathcal{N}_{2}}\right) \alpha_{2} K_{2} \\
& =\frac{\beta_{2, h m}}{\mu_{2, m}} \frac{S_{m, 2}^{0}}{N_{2, h}^{0}}
\end{aligned}
$$

We derive the Jacobian of our system at 0

$$
J^{\prime}(0)=\left(\begin{array}{cccc}
0 & 0 & F_{1} & F_{2} \\
0 & 0 & F_{3} & F_{4} \\
g_{1, h m}^{\prime} & 0 & 0 & 0 \\
0 & g_{2, h m}^{\prime} & 0 & 0
\end{array}\right)
$$

where

$$
\begin{aligned}
& F_{1}=\frac{\left(\eta_{h}+\mu_{h}+\gamma_{2} m_{12}\right) \beta_{1, m h}}{\left(\eta_{h}+\mu_{h}\right)\left(\eta_{h}+\mu_{h}+\gamma_{1} m_{21}+\gamma_{2} m_{12}\right)}, \\
& F_{2}=\frac{\gamma_{2} m_{12} \beta_{2, m h}}{\left(\eta_{h}+\mu_{h}\right)\left(\eta_{h}+\mu_{h}+\gamma_{1} m_{21}+\gamma_{2} m_{12}\right)}, \\
& F_{3}=\frac{\gamma_{1} m_{21} \beta_{1, m h}}{\left(\eta_{h}+\mu_{h}\right)\left(\eta_{h}+\mu_{h}+\gamma_{1} m_{21}+\gamma_{2} m_{12}\right)} \\
& F_{4}=\frac{\left(\eta_{h}+\mu_{h}+\gamma_{1} m_{21}\right) \beta_{2, m h}}{\left(\eta_{h}+\mu_{h}\right)\left(\eta_{h}+\mu_{h}+\gamma_{1} m_{21}+\gamma_{2} m_{12}\right)}
\end{aligned}
$$

$$
\begin{aligned}
& p(\lambda)=\lambda^{4}-\frac{1}{\eta_{h}+\mu_{h}+\gamma_{1} m_{21}+\gamma_{2} m_{12}} \\
& \times\left(\left(\eta_{h}+\mu_{h}+\gamma_{1} m_{21}\right) \mathcal{R}_{0,2}^{2}+\left(\eta_{h}+\mu_{h}+\gamma_{2} m_{12}\right) \mathcal{R}_{0,1}^{2}\right) \lambda^{2}
\end{aligned}
$$$$
\left.\times\left(\eta_{h}+\mu_{h}+\gamma_{1} m_{21}\right) \mathcal{R}_{0,2}^{2} \mathcal{R}_{0,1}^{2}-\gamma_{2} m_{21} \gamma_{1} m_{12} \mathcal{R}_{0,2}^{2} \mathcal{R}_{0,1}^{2}\right) .
$$

Thus setting $x=\lambda^{2}$, we obtain the following a second order polynomial

$$
\begin{aligned}
& p(x)=x^{2}-\frac{1}{\eta_{h}+\mu_{h}+\gamma_{1} m_{21}+\gamma_{2} m_{12}} \\
& \left(\left(\eta_{h}+\mu_{h}+\gamma_{1} m_{21}\right) \mathcal{R}_{0,2}^{2}+\left(\eta_{h}+\mu_{h}+\gamma_{2} m_{12}\right) \mathcal{R}_{0,1}^{2}\right) x \\
& +\left(\frac{1}{\eta_{h}+\mu_{h}+\gamma_{1} m_{21}+\gamma_{2} m_{12}}\right)^{2}\left(\left(\eta_{h}+\mu_{h}+\gamma_{2} m_{12}\right)\right. \\
& \left.\times\left(\eta_{h}+\mu_{h}+\gamma_{1} m_{21}\right) \mathcal{R}_{0,2}^{2} \mathcal{R}_{0,1}^{2}-\gamma_{2} m_{21} \gamma_{1} m_{12} \mathcal{R}_{0,2}^{2} \mathcal{R}_{0,1}^{2}\right) .
\end{aligned}
$$

Then

$$
\begin{aligned}
& p(x)=\left(x-\frac{1}{2} \frac{1}{\eta_{h}+\mu_{h}+\gamma_{1} m_{21}+\gamma_{2} m_{12}}\right. \\
& \left.\times\left(\left(\eta_{h}+\mu_{h}+\gamma_{1} m_{21}\right) \mathcal{R}_{0,2}^{2}+\left(\eta_{h}+\mu_{h}+\gamma_{2} m_{12}\right) \mathcal{R}_{0,1}^{2}\right)\right)^{2} \\
& -\frac{1}{4}\left(\frac{1}{\eta_{h}+\mu_{h}+\gamma_{1} m_{21}+\gamma_{2} m_{12}}\right)^{2} \\
& \times\left(\left(\left(\eta_{h}+\mu_{h}+\gamma_{1} m_{21}\right) \mathcal{R}_{0,2}^{2}+\left(\eta_{h}+\mu_{h}+\gamma_{2} m_{12}\right) \mathcal{R}_{0,1}^{2}\right)^{2}\right. \\
& +4 \gamma_{2} m_{21} \gamma_{1} m_{12} \mathcal{R}_{0,2}^{2} \mathcal{R}_{0,1}^{2} .
\end{aligned}
$$

Thus

$$
\begin{aligned}
& p(x)=\left(x-\frac{1}{2} \frac{1}{\eta_{h}+\mu_{h}+\gamma_{1} m_{21}+\gamma_{2} m_{12}} \times\right. \\
& \left.\left(k_{2 h} \mathcal{R}_{0,2}^{2}+k_{1 h} \mathcal{R}_{0,1}^{2}-\sqrt{Z(\gamma)}\right)\right) \\
& \times\left(x-\frac{1}{2} \frac{1}{\eta_{h}+\mu_{h}+\gamma_{1} m_{21}+\gamma_{2} m_{12}}\right. \\
& \left.\left(k_{2 h} \mathcal{R}_{0,2}^{2}+k_{1 h} \mathcal{R}_{0,1}^{2}+\sqrt{Z(\gamma)}\right)\right),
\end{aligned}
$$

with

$$
\begin{aligned}
& Z(\gamma)=\left(k_{2 h} \mathcal{R}_{0,2}^{2}-k_{1 h} \mathcal{R}_{0,1}^{2}\right)^{2}+4 \gamma_{2} m_{12} \gamma_{1} m_{21} \mathcal{R}_{0,2}^{2} \mathcal{R}_{0,1}^{2}>0 \\
& k_{1 h}=\left(\eta_{h}+\mu_{h}+\gamma_{2} m_{12}\right) \\
& k_{2 h}=\left(\eta_{h}+\mu_{h}+\gamma_{1} m_{21}\right)
\end{aligned}
$$


Thus, we deduce the following exact formula for $\mathcal{R}_{0}^{2}$ :

$$
\begin{aligned}
& \mathcal{R}_{0}^{2}=\frac{1}{2} \frac{1}{\eta_{h}+\mu_{h}+\gamma_{1} m_{21}+\gamma_{2} m_{12}} \\
& \times\left(k_{2 h} \mathcal{R}_{0,2}^{2}+k_{1 h} \mathcal{R}_{0,1}^{2}+\sqrt{Z(\gamma)}\right) .
\end{aligned}
$$

When $\gamma_{1}=\gamma_{2}=0$, we immediately have

$$
\mathcal{R}_{0}^{2}=\frac{1}{2}\left(\mathcal{R}_{0,2}^{2}+\mathcal{R}_{0,1}^{2}+\left|\mathcal{R}_{0,2}^{2}-\mathcal{R}_{0,1}^{2}\right|\right),
$$

which is equivalent to $\mathcal{R}_{0}^{2}=\max \left(\mathcal{R}_{0,1}^{2}, \mathcal{R}_{0,2}^{2}\right)$.

Moreover, if $\mathcal{R}_{0,1}^{2}=\mathcal{R}_{0,2}^{2}$, then $\mathcal{R}_{0}^{2}=\mathcal{R}_{0,1}^{2}=\mathcal{R}_{0,2}^{2}$ whatever the migration matrix is.

Then, we will consider various migration matrix between city 1 and city 2 , to show how different can be the results according to the movement rates....

Example 1: The numerical simulations presented here have been obtained using nonstandard finite difference scheme (see [1], [2], [3], [4], [6] for an overview on nonstandard methods and applications to biological systems). We consider parameters (see table $\nabla$ in Appendix B) such that $\mathcal{R}_{0,1}>1$ and $\mathcal{R}_{0,2}<1$, i.e.

\begin{tabular}{|lllllll|}
\hline & $N_{1, h}$ & $K_{1}$ & $\mathcal{R}_{0,1}$ & $N_{2, h}$ & $K_{2}$ & $\mathcal{R}_{0,2}$ \\
\hline Case 1 & $8.10^{4}$ & $3 N_{1, h}$ & 1.3017 & $8.10^{4}$ & $N_{2, h}$ & 0.434 \\
\hline Case 2 & $8.10^{4}$ & $3 N_{1, h}$ & 1.3017 & $10^{4}$ & $N_{2, h}$ & 0.434 \\
\hline Case 3 & $10^{4}$ & $3 N_{1, h}$ & 1.3017 & $8.10^{4}$ & $N_{2, h}$ & 0.434 \\
\hline
\end{tabular}

Thus, in order to keep the total population $N_{1}+N_{2}$ constant, we have many choices for the migration matrix. Let us first consider simply

$$
\mathcal{M}=M_{c}\left(\begin{array}{cc}
-1 & \frac{N_{1, h}}{N_{2, h}} \\
1 & -\frac{N_{1, h}}{N_{2, h}}
\end{array}\right),
$$

where $M_{c}$ is a positive constant. We would like to study the time evolution of the disease with respect to $\Gamma=$ $\operatorname{diag}\left(\gamma_{1}, \gamma_{2}\right)$, taking into account the movement rates and different values for $M_{c}$.

- Case 1. We consider $M_{c}=1$ and cities with the same populations, except for the larvae capacity such that the we obtain distinct basic reproduction numbers. In Fig. 2] we consider $\gamma_{1}=\gamma_{2}$ : in that case, when $\gamma>0$, the epidemic occurs in both cities.

If we consider $\gamma_{1}=1$ and $\gamma_{2}=0.5$, then the dynamic change: if less infected people from city 2 are able to move to city 1 , i.e. $\gamma_{2}$ small, then the general basic reproduction number decreases rapidly under 1 . This is confirmed In Fig. 4 where we show $\mathcal{R}_{0}$ with respect to $\gamma_{1}$ and $\gamma_{2}$ : it clearly
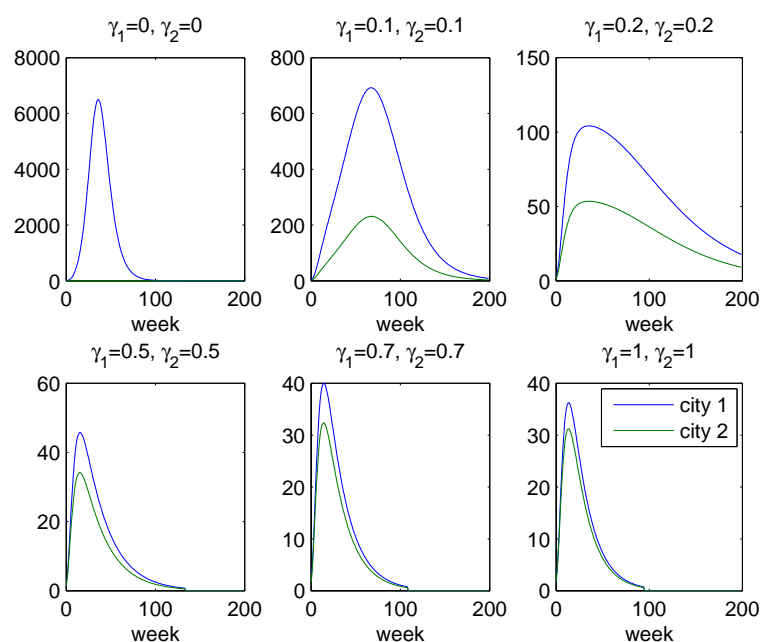

Fig. 2. Simulation of the evolution of the infected population per week for different values of $\gamma=\gamma_{1}=\gamma_{2}$.

indicates that small values for $\gamma_{2}$ help to decrease the epidemiological risk. In fact, following Fig. 4 the best combination being $\Gamma=\operatorname{diag}(1,0)$. This is due to the fact that city 1 has the largest basic reproduction number: thus movement of infected individuals from city 1 to city 2 , reduce the number of infected people in city 1 , and thus the risk of propagation. The model behaves like a quarantine model.
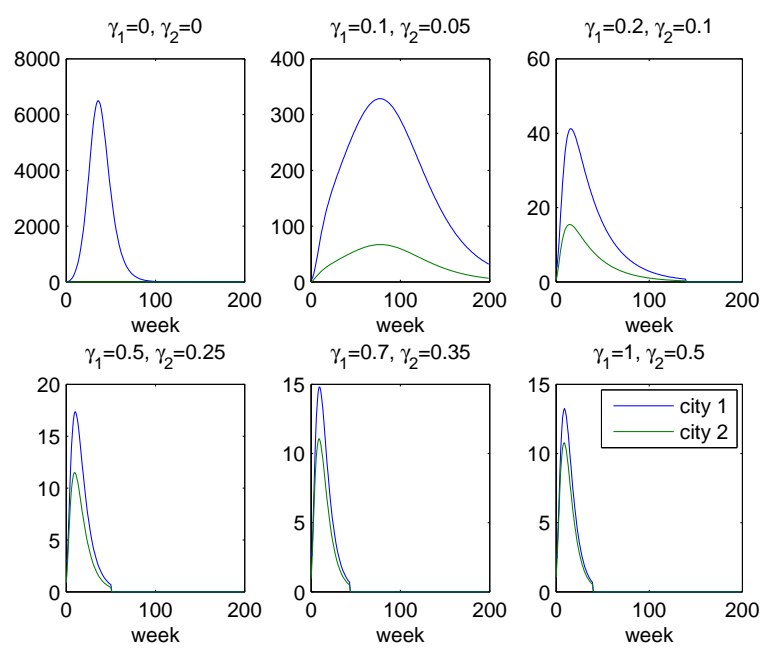

Fig. 3. Simulation of the evolution of the infected population per week for different values of $\Gamma$.

- Case 2. We consider $M_{c}=1$. Numerical simulations are presented in Fig. 5 for different values of $\gamma$. Of course without Human movements, the first city faces a huge epidemic while in the second noth- 


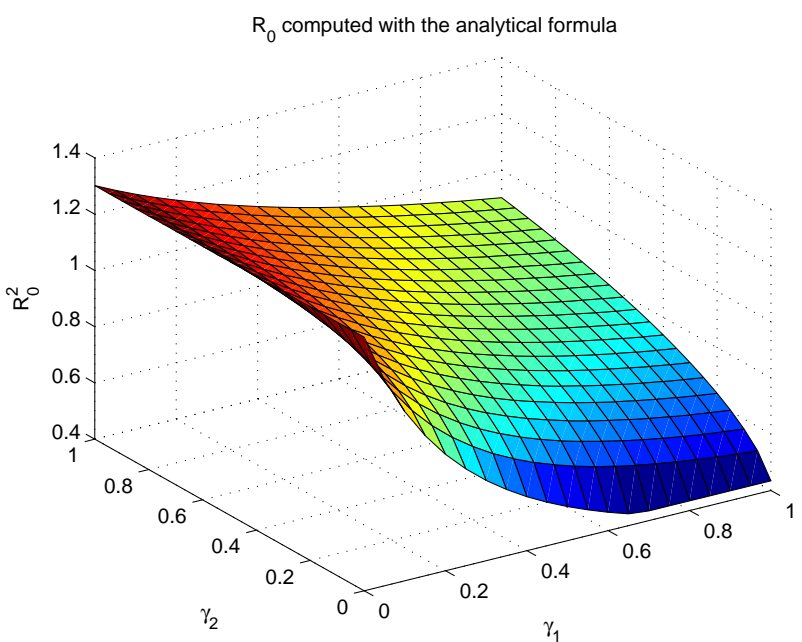

Fig. 4. Evolution of $\mathcal{R}_{0}^{2}$ with respect to $\gamma_{1}$ and $\gamma_{2}$

ing occurs. For $\gamma>0$, then the dynamic changes drastically: in particular in city 1 , we observe a decay in the Number of Infected people as soon as $\gamma>$, while in city 2 , an outbreak appears. This is confirmed in Fig, 6, where $\mathcal{R}_{0}^{2}$ has been computed with the exact formula (22): as long as $\gamma_{21}>0$, $\mathcal{R}_{0}>1$, which indicates that an epidemic will occur in all cities.

In this case, city 1 , where $\mathcal{R}_{0}^{2}>1$, has a large population compared to city 2 . Thus the flow of infected people from city 1 to city 2 , will increase the number of infected people in city 2 , as long as the epidemic occurs in city 2 . Thus disease control is necessary in city 1 in order to avoid the risk of disease spreading. In particular, reducing local and large $\mathcal{R}_{0, i}^{2}$ could be helpfull to lower the general basic reproduction number, but this may not be not necesserally sufficient or, even, possible. In this case, city 1, with the largest population, has the strongest impact on $\mathcal{R}_{0}^{2}$ : compare also with Fig. 4 where both cities have the same population.

Even if $M_{c}$ decays this will not change the overall behavior: $\mathcal{R}_{0}$ will stay greater than one. In fact decaying $M_{c}$ is like decaying $\gamma$ and it is obvious that for small values of $\gamma$ human movements implies a spreding of the disease in all patches

- Case 3. We first consider $M_{c}=1$. Numerical simulations are presented in Fig. 7 Contrary to case 1, city 2 has now the largest population with $\mathcal{R}_{0,2}<1$. Thus without migration, only city 1 is impacted by a huge epidemic, but, as soon as $\gamma_{1}>0$, the number of infected cases reduce drastically in city 1 , while
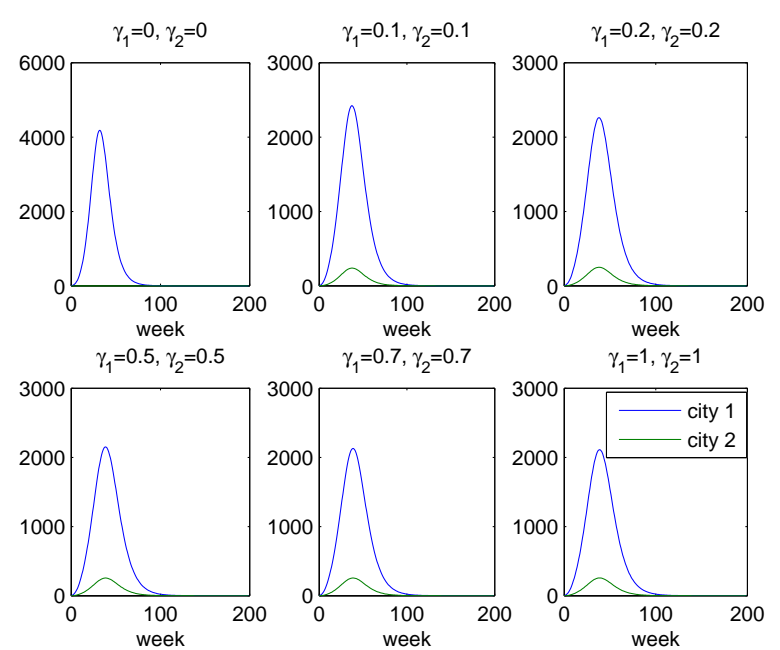

Fig. 5. Simulation of the evolution of the infected population per week for different values of $\gamma$.

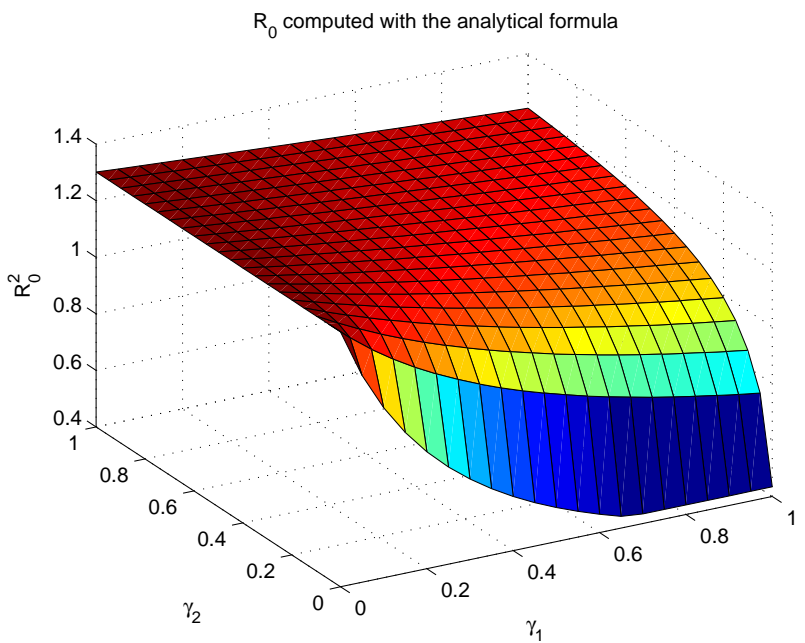

Fig. 6. Evolution of $\mathcal{R}_{0}^{2}$ with respect to $\gamma_{1}$ and $\gamma_{2}$

in city 2 a small outbreak appear. Finally, as $\gamma_{1}$ increases, the epidemic becomes a small outbreak for both cities (see Fig 8). Thus, the city with the largest population and a local basic reproduction number less than one has a positive impact on the general basic reproduction number: compare Fig 8 with Fig 6

Indeed, if we consider $M_{c}=0.1$, for instance, then the dynamic is completely different and shows that the disease will spread in all patches (see Figs. (9) but as $\gamma$ increases the force of the disease decays, which is confirmed by the computation of the basic reproduction number in Fig. 10 In fact this computation is equivalent to consider $\gamma_{i} \in[0,0.1]$ 

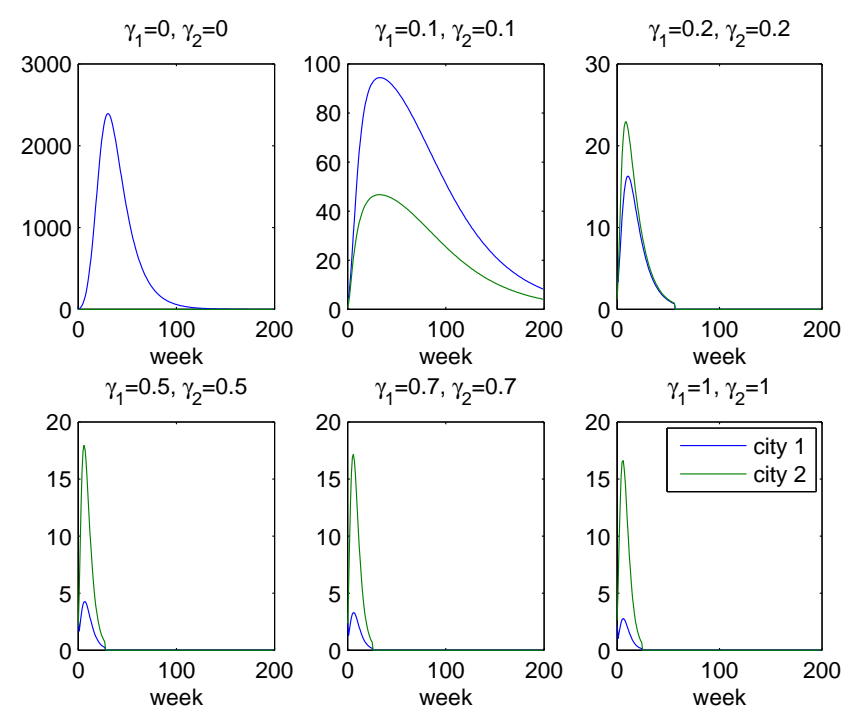

Fig. 7. Simulation of the evolution of the infected population per week for different values of $\gamma$.

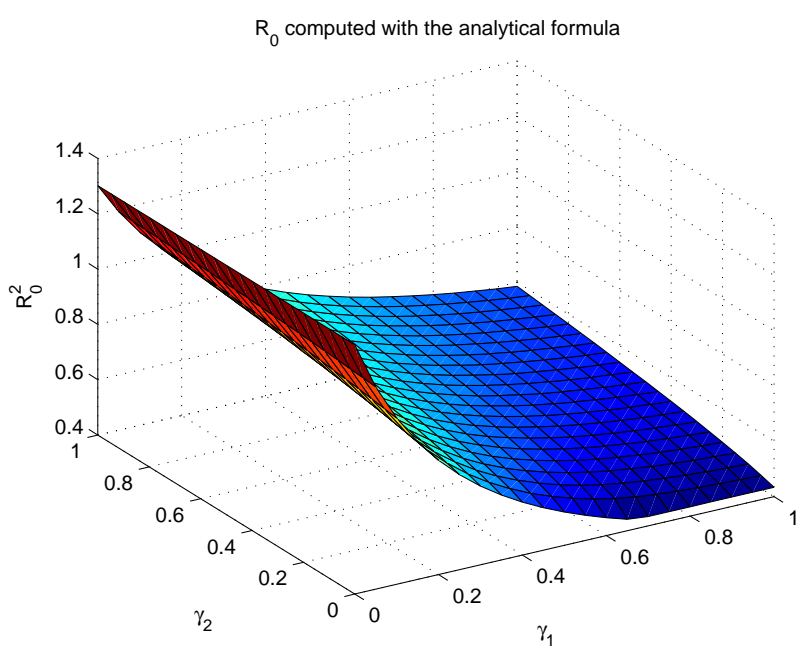

Fig. 8. Evolution of $\mathcal{R}_{0}^{2}$ with respect to $\gamma_{1}$ and $\gamma_{2}$

when $M_{c}=1$.

Thus, in case 3, at least, vector control can be necessary or not, depending on the movements matrice: when $M_{c}=1$, the disease will disappear naturally... while, not when $M_{c}=0.1$.

In any case, it is clear that Human movements may have a benefit effect when the largest city has a basic reproduction number lower than 1 .

Our example emphasizes the importance of the migration (movement) matrice and how important is the construction of such a matrice to understand or to well capture the whole dynamic. And this is only a in 2-patches model!
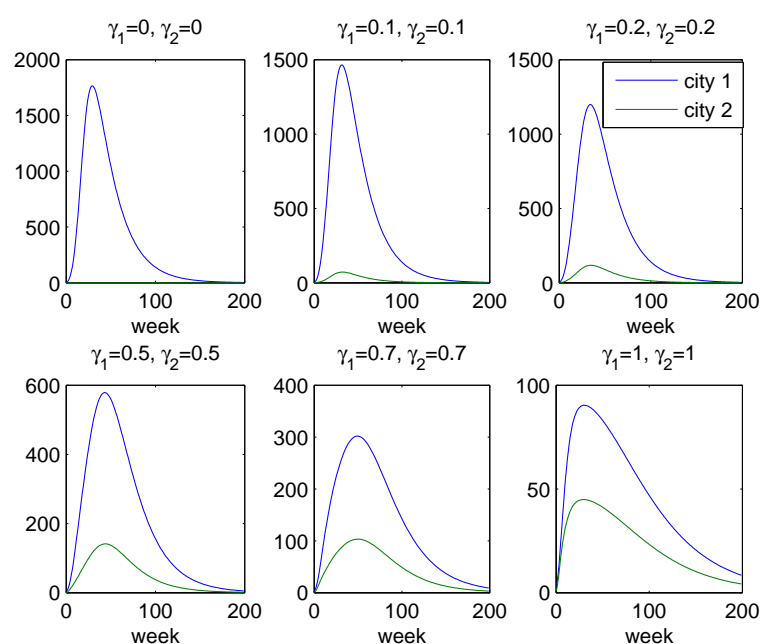

Fig. 9. Simulation of the evolution of the infected population per week for different values of $\gamma$ and $M_{c}=0.1$.

$\mathrm{R}_{0}$ computed with the analytical formula

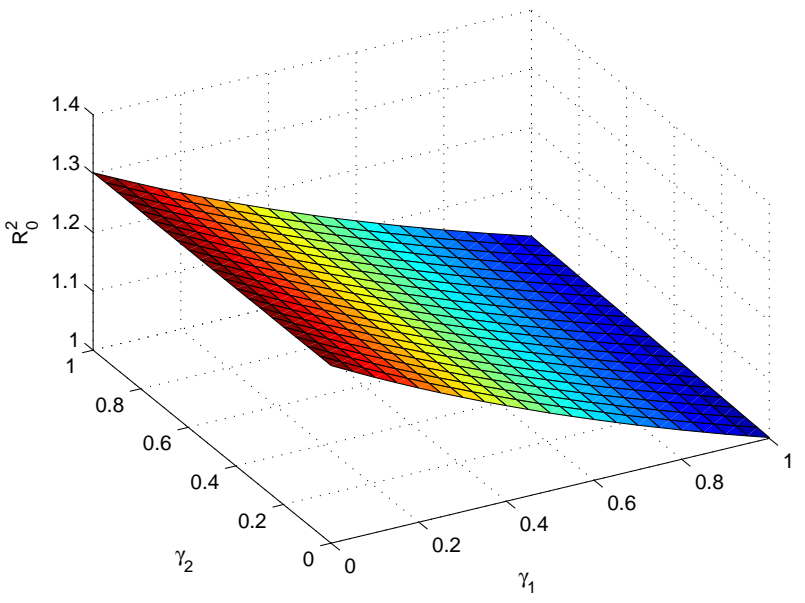

Fig. 10. Evolution of $\mathcal{R}_{0}^{2}$ with respect to $\gamma_{1}$ and $\gamma_{2}$, when $M_{c}=0.1$.

In fact we can go further with this example and for instance, consider another movement matrix:

$$
\mathcal{M}=M_{c}\left(\begin{array}{cc}
-\frac{N_{1, h}}{N_{2, h}} & 1 \\
\frac{N_{1, h}}{N_{2, h}} & -1
\end{array}\right)
$$

with $M_{c}=1$. Let us first consider case 2: compare Figs. 11 and $6 \mathcal{R}_{0}^{2}$ is not the same, but, following Fig. 11] it seems that human movements have limited impacts on $\mathcal{R}_{0}^{2}$. This example clearly shows that if locally the basic reproduction number is lower than 1 then the impact of the disease will be limited, even if there infected people moves from city 1 .

In case 3 , in contrary, the new movement matrix changes drastically the behavior of the basic reproduction number: compare Figs. 12 and 8 Here, the impact of 
the largest city (city 2 ) is really important. In that case, even if $\gamma$ is small, $\mathcal{R}_{0}^{2}$ decays rapidly and the disease dies out.

Thus depending on $\mathcal{M}, \mathcal{R}_{0}^{2}$ can be very different, which may imply unappropriate control decisions. This clearly indicates the importance of the construction of the movement Matrices.

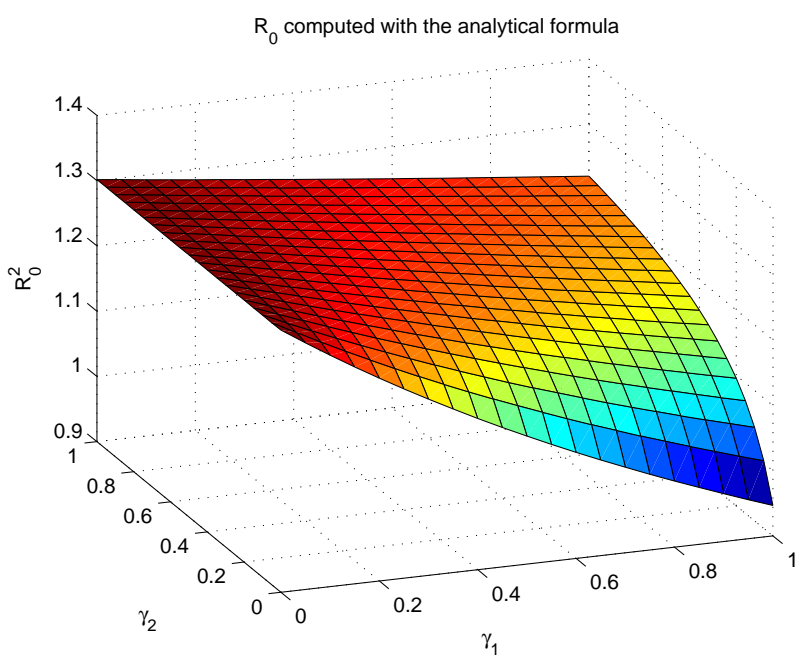

Fig. 11. Evolution of $\mathcal{R}_{0}^{2}$ with respect to $\gamma_{1}$ and $\gamma_{2}$

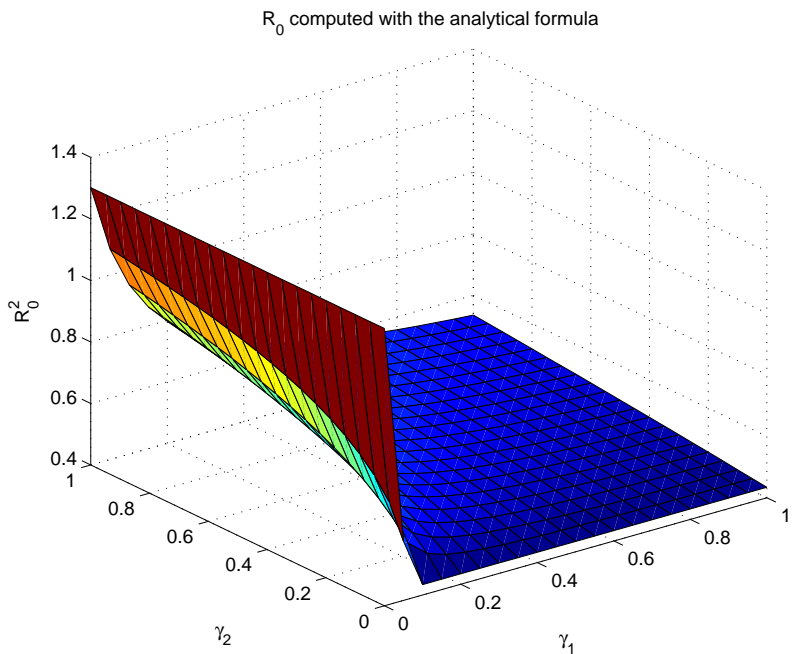

Fig. 12. Evolution of $\mathcal{R}_{0}^{2}$ with respect to $\gamma_{1}$ and $\gamma_{2}$

\section{Conclusion}

Our study show how complex it is to take into account human movements in a vector-borne disease model. More complex is the network and more difficult it will be to control the spreading of the disease. In fact, we say that our approach permit to sustain or to establish that local interventions can be benefit for the whole population. Mathematically the model is not very easy to handle, but we have been able to show some interesting results. In particular, we showed the link between the general basic reproduction number and local ones, which is really important from a practical point of view. Indeed, our illustrative examples indicate that measuring or estimating local basic reproductive numbers is of major importance not only to map the epidemiological risk in order to take into account where the risk of an epidemic is high, but to be able to indicates priority to lower some local basic reproduction numbers. Thus, among all cities where the risk is high, it seems important to make vector control in priority in cities where the Human population is large. In any case, each city has to make appropriate vector control campaign to lower the epidemiological risk. In the case where some cities, with the largest populations, may have for any reason large basic reproduction numbers, then the disease can spread quickly to the whole domain, even when $\Gamma$ is small, according to the network. In contrary when only small cities have large local basic reproduction numbers, human movements can have a benefit effect, i.e. the disease dies out.

Of course, the model can still be improved in different ways. It might be interesting to consider a variable total population and/or time-dependant parameters [17]. But, for a practical use in Réunion Island, a first step would be to build the right movements matrix between cities, using precise human movements data. Finally, this model could be adapted to link several islands (Mauritius, La Réunion, Comoros and Madagascar) located in the Indian Ocean.

\section{ACKNOWLEDGMENT}

This study was funded by the French Ministry of Health and the European Regional Development Fund (ERDF) within the "SIT feasibility programme" in Réunion Island. The authors would like to thank Guy Lemperiere (IRD, coordinator of the SIT project in Réunion island), Louis-Clément Gouagna (IRD), Jérémie Gilles and Clélia Oliva (AIEA, Seibersdorf, Austria), Sébastien Boyer (IRD), and Jean-Sebastien Dehecq (ARS, Réunion island), for stimulating discussions. YD is also grateful to Didier Fontenille (IRD, head of the SIT project) and Koussay Dellagi (CRVOI, Réunion island) for their support.

The authors would like to thank the reviewers for their constructive comments that helped to improve the manuscript. 
AMAP (Botany and Computational Plant Architecture) is a joint research unit which associates CIRAD (UMR51), CNRS (UMR5120), INRA (UMR931), IRD (2M123), and Montpellier 2 University (UM27); http://amap.cirad.fr/

\section{REFERENCES}

[1] R. Anguelov, Y. Dumont, J.M-S. Lubuma and M. Shillor, Dynamically consistent non-standard finite difference schemes for the MSEIR epidemiological model, In: T. Simos, G. Psihoyios, Ch Tsitouras (eds), Proceedings of the International Conference of Numerical Analysis and Applied Mathematics, Crete, Greece, 18-22 September 2009, American Institute of Physics Conference Proceedings-AIP 1168, Volume 2 (2009), 1213-1216.

[2] R. Anguelov, Y. Dumont, J.M-S. Lubuma and M. Shillor, Comparison of some standard and nonstandard numerical methods for the MSEIR epidemiological model, Proceedings of the International Conference of Numerical Analysis and Applied Mathematics, Crete, Greece, 18-22 September 2009, American Institute of Physics Conference Proceedings-AIP 1168, Volume 2 (2009), 1209-1212.

[3] R. Anguelov,Y. Dumont, J.M.-S. Lubuma, and M. Shillor, M., Dynamically consistent nonstandard finite difference schemes for epidemiological models, Journal of Computational and Applied Mathematics 255 (2014), 161-182. http://dx.doi.org/10.1016/j.cam.2013.04.042

[4] R. Anguelov, Y. Dumont, J. Lubuma and E. Mureithi, Stability Analysis and Dynamics Preserving Non-Standard Finite Difference Schemes for a Malaria Model, Mathematical Population Studies 20 (2) (2013), 101-122. http://dx.doi.org/10.1080/08898480.2013.777240

[5] R. Anguelov, Y. Dumont, and J.M-S. Lubuma, Mathematical Modeling of Sterile Insect Technology for Control of Anopheles Mosquito, Computers \& Mathematics with Applications 64 (2012), 374-389. http://dx.doi.org/10.1016/j.camwa.2012.02.068

[6] R. Anguelov, Y. Dumont, and J.M-S. Lubuma, On Nonstandard Finite Difference Schemes in Biosciences, AMITANS 2012, june 2012, AIP Conf. Proc. 1487, 212-223.

[7] J. Arino, Diseases in metapopulations. Modeling and dynamics of infectious diseases, Ser. Contemp. Appl. Math. CAM, 11, Higher Ed. Press, Beijing, 2009, 64-122.

[8] P. Auger, E. Kouokam, G. Sallet, M. Tchuente, and B. Tsanou, The Ross-Macdonald model in a patchy environment, Mathematical Biosciences 216 (2008), 123-131. http://dx.doi.org/10.1016/j.mbs.2008.08.010

[9] A. Berman and R.J. Plemmons, Nonnegative matrices in the Mathematical Sciences, SIAM, Classic in Applied Mathematics, Philadelphia 1994, 361 pages.

[10] C. Castillo-Chavez, S. Blower, P. van den Driessche, D. Kirschner, A-A. Yakubu; Mathematical Approaches for Emerging and Re-emerging Infectious Diseases : An introduction, volume 125 of IMA. Springer Verlag Berlin Heidelberg, New York, 2002.

[11] C. Castillo-Chavez, S. Blower, P. van den Driessche, D. Kirschner, A-A. Yakubu; Mathematical Approaches for Emerging and Re-emerging Infectious Diseases : Part II : Models, Methods and Theory. Springer-Verlag Berlin-Heidelberg-New York, 2002.
[12] H. Delatte, G. Gimonneau, A. Triboire and D. Fontenille, Influence of temperature on immature development, survival, longevity, fecundity and gonotrophic cycles of Aedes albopictus (Skuse), vector of Chikungunya and dengue in the Indian Ocean, J Med Entomol 46 (2009) 33-41. http://dx.doi.org/10.1603/033.046.0105

[13] H. Delatte, C. Paupy, J.S. Dehecq, J.Thiria, A. B. Failloux and D. Fontenille, Aedes albopictus, vector of Chikungunya and dengue viruses in Réunion Island: biology and control, Parasite 15 (1) (2008), 3-13. http://dx.doi.org/10.1051/parasite/2008151003

[14] H. Delatte, J.S. Dehecq, J. Thiria, C. Domerg, C. Paupy and D. Fontenille, Geographic Distribution and Developmental Sites of Aedes albopictus (Diptera: Culicidae) during a Chikungunya Epidemic Event, Vector Borne Zoonotic Dis 8 (2008), 25-34. http://dx.doi.org/10.1089/vbz.2007.0649

[15] M. Dubrulle, L. Mousson, S. Moutailler, M. Vazeille, A.-B. Failloux, Chikungunya virus and Aedes mosquitoes: Saliva is infectious as soon as two days after oral infection. PLoS One 4(6) (2009). http://dx.doi.org/10.1371/journal.pone.0005895

[16] C. Dufourd and Y. Dumont, Modeling and Simulations of mosquito dispersal. The case of Aedes albopictus, Biomath 1 (2), 2012. http://dx.doi.org/10.11145/j.biomath.2012.09.262

[17] C. Dufourd and Y. Dumont, Impact of environmental factors on mosquito dispersal in the prospect of Sterile Insect Technique control, Computer \& Mathematics with Applications. In press. http://dx.doi.org/10.1016/j.camwa.2013.03.024

[18] Y. Dumont, F. Chiroleu and C. Domerg, On a temporal model for the Chikungunya disease: modeling, theory and numerics, Mathematical Biosciences 213 (2008), 70-81. http://dx.doi.org/10.1016/j.mbs.2008.02.008

[19] Y. Dumont and F. Chiroleu, Vector Control for the Chikungunya Disease, Mathematical Bioscience and Engineering, 7-2 (2010), 315-348.

[20] Y. Dumont, Modeling Mosquito distribution. Impact of the Vegetation. In Simos, T., Psihoyios, G., Tsitouras, C., Anastassi, Z. (Eds) Proceedings of the International Conference of Numerical Analysis and Applied Mathematics, Haldiki, Greece, 19-25 September 2011. Haldiki. American Institute of Physics Conference Proceedings-AIP 1389, 2011, 1244-1247.

[21] Y. Dumont, C. Dufourd, Spatio-temporal modeling of Mosquito distribution. AIP conference proceedings , 1404 (1) (2011): $162-$ 167. http://dx.doi.org/10.1063/1.3659916

[22] Y. Dumont and J.M. Tchuenche, The Sterile Insect Technique for the Chikungunya and Aedes albopictus, Journal of Mathematical Biology, 65 (5) (2012), 809-854. http://dx.doi.org/10.1007/s00285-011-0477-6

[23] D. Gao and S. Ruan, A Multipatch Malaria Model with Logistic Growth Populations, SIAM Journal on Applied Mathematics 72 (3) (2012), 819-841. http://dx.doi.org/10.1137/110850761

[24] H.W. Hethcote, H.R. Thieme, Stability of the endemic equilibrium in epidemic models with subpopulations, Math. Biosci. 75 (2) (1985), 205-227. http://dx.doi.org/10.1016/0025-5564(85)90038-0

[25] MJ Keeling, ON Bjornstad, BT Grenfell. Metapopulation Dynamics of Infectious Diseases in Metapopulation Dynamics, I Hanski, OE Gaggiotti (ed.), Elsevier Press (2003), 415-446.

[26] V. Lakshmikantham, S. Leela, A.A. Martynyuk, Stability Analysis of Nonlinear Systems. Dekker, New York (1989). 
[27] E. Martin, S. Moutailler, Y. Madec, and A.-B. Failloux, Differential responses of the mosquito Aedes albopictus from the Indian Ocean region to two chikungunya isolates, BMC Ecol. 10:8 (2010). http://dx.doi.org/10.1186/1472-6785-10-8

[28] R.E. Mickens, Nonstandard finite difference models of differential equations, World Scientific, Singapore, (1994).

[29] D. Moulay, M.A. Aziz-Alaoui, and M. Cadivel (2011) The Chikungunya disease: modeling, vector and transmission global dynamics, Math. Biosc. 229 (1): 60-63 http://dx.doi.org/10.1016/j.mbs.2010.10.008

[30] G. Pialoux, B-A. Gaüzere, S. Jauréguiberry and M. Strobel, Chikungunya, an epidemic arbovirosis, Lancet Infectious Dis., 7 (2007), 319-327. http://dx.doi.org/10.1016/S1473-3099(07)70107-X

[31] M.-K. Soumahoro, P. Gérardin, P.Y. Boelle, J. Perrau, A. Fianu, et al., Impact of Chikungunya Virus Infection on Health Status and Quality of Life: A Retrospective Cohort Study. PLoS ONE 4(11): e7800 (2009). http://dx.doi.org/10.1371/journal.pone.0007800

[32] J.E. Staples, R.F. Breiman, and A.M. Powers, Chikungunya Fever: An Epidemiological Review of a Re-Emerging Infectious Disease, Clinical Infectious Diseases Vol (6) (2009), 942-948.

[33] P. van den Driessche and J. Watmough, Reproduction numbers and sub-threshold endemic equilibria for compartmental models of disease transmission, Mathematical Biosciences 180 (2002), $29-48$.

http://dx.doi.org/10.1016/S0025-5564(02)00108-6

[34] M. Vazeille, S. Moutailler, D. Coudrier, C. Rousseaux, H. Khun, M. Huerre, J. Thiria, J.S. Dehecq, D. Fontenille, I. Schuffenecker, P. Desprez and A.-B. Failloux, Two Chikungunya isolates from the outbreak of la Réunion (Indian Ocean), exhibit different patterns of infection in the mosquito, Aedes albopictus. PLoS One 2, 11 (2007).

http://dx.doi.org/10.1371/journal.pone.0001168

[35] M. Vazeille, L. Mousson and A.-B. Failloux, Failure to demonstrate experimental vertical transmission of the epidemic strain of Chikungunya virus in Aedes albopictus from La Réunion Island, Indian Ocean. Mem. Inst. Oswaldo Cruz, Rio de Janeiro, 104 (4) (2009), 632-635.

[36] M. Vidyasagar, Decomposition techniques for large-scale systems with nonadditive interactions: stability and stabilization. IEEE Trans. Autom. Control 25, 773 (1980). http://dx.doi.org/10.1109/TAC.1980.1102422

\section{APPENDIX A: A USEFUl THEOREM}

For reader's convenience, we recall the following useful theorem, proved in [5].

Consider the following cooperative system of ODEs

$$
\frac{d x}{d t}=g(x),
$$

where $D \subseteq \mathbb{R}^{n}$ and $g: D \rightarrow \mathbb{R}^{n}$ is continuous. It is assumed that $D \subset$ closure(interior $(D))$ and that for some $\delta>0$ the vector fields defined by $g(t, \cdot), t \in$ $[0, \delta)$, are all directed inwards at the points of $\partial D$. This is enough to ensure that for every a $\in D$ there exists $T_{a}>0$ such that the system (23) has a solution $x(\mathbf{a}, t)$ on the interval $\left[0, T_{a}\right)$ which satisfies $x(\mathbf{a}, 0)=\mathbf{a}$. We further assume that $g$ is such that the solution initiated at a is unique. We also assume that $\left[0, T_{a}\right)$ is the maximal (nonnegative) interval of existence of $x(\mathbf{a}, t)$.

Let $\mathbf{a}, \mathbf{b} \in \mathbb{R}^{n}$ with $\mathbf{a} \leq \mathbf{b}$, and

$$
[\mathbf{a}, \mathbf{b}]=\left\{x \in \mathbb{R}^{n}: \mathbf{a} \leq x \leq \mathbf{b}\right\} .
$$

Theorem 5 ([[5]): Let $\mathbf{a}, \mathbf{b} \in D$ be such that $\mathbf{a}<\mathbf{b}$, $[\mathbf{a}, \mathbf{b}] \subseteq D$ and $g(\mathbf{b}) \leq \mathbf{0} \leq g(\mathbf{a})$. Then (23) defines a (positive) dynamical system on $[\mathbf{a}, \mathbf{b}]$. Moreover, if $[\mathbf{a}, \mathbf{b}]$ contains a unique equilibrium $p$ then $p$ is globally asymptotically stable on $[\mathbf{a}, \mathbf{b}]$.

\section{APPENDIX B: BIOLOGICAL PARAMETERS}

\begin{tabular}{|c|c|}
\hline Parameters Description & value \\
\hline$\beta_{m h}$ ( Transmission probability from $I_{m}$ (per bite) $)$ & 0.365 \\
\hline$\beta_{h m}\left(\right.$ Transmission probability from $I_{h}$ (per bite) $)$ & 0.365 \\
\hline $1 / \mu_{h}$ (Average lifespan of humans (in days) $)$ & $78 \times 365$ \\
\hline $1 / \eta_{h}$ ( Average viremic period (in days) & 3 \\
\hline $1 / \eta_{m}$ ( Extrinsic incubation period (in days) $)$ & 2 \\
\hline $1 / \mu_{m}$ (Average lifespan of female mosquitoes (in days)) & 11 \\
\hline$\mu_{b}$ (Nb of eggs at each deposit per capita (per day) $)$ & 7 \\
\hline$\mu_{A}$ (Natural mortality of larvae (per day)) & 2 \\
\hline$\eta_{A}$ (Maturation rate from larvae to adult (per day)) & $\approx 0.05$ \\
\hline
\end{tabular}

